\title{
ON SYMPLECTIC FILLINGS OF LENS SPACES
}

\author{
PAOLO LISCA
}

\begin{abstract}
Let $\bar{\xi}_{\text {st }}$ be the contact structure naturally induced on the lens space $L(p, q)=S^{3} / \mathbb{Z} / p \mathbb{Z}$ by the standard contact structure $\xi_{\text {st }}$ on the threesphere $S^{3}$. We obtain a complete classification of the symplectic fillings of $\left(L(p, q), \bar{\xi}_{\mathrm{st}}\right)$ up to orientation-preserving diffeomorphisms. In view of our results, we formulate a conjecture on the diffeomorphism types of the smoothings of complex two-dimensional cyclic quotient singularities.
\end{abstract}

\section{INTRODUCTION AND STATEMENT OF RESULtS}

A (coorientable) contact three-manifold is a pair $(Y, \xi)$, where $Y$ is a closed three-manifold and $\xi \subset T Y$ a two-dimensional distribution given as the kernel of a one-form $\alpha \in \Omega^{1}(Y)$ such that $\alpha \wedge d \alpha$ is a volume form. The orientation on $Y$ determined by $\alpha \wedge d \alpha$ only depends on the distribution $\xi$. We shall always assume that the underlying manifold of a contact three-manifold is oriented, and that the orientation is the one induced by the contact structure.

A symplectic filling of a closed contact three-manifold $(Y, \xi)$ is the pair $(X, \omega)$ consisting of a smooth, compact, connected four-manifold $X$ and a symplectic form $\omega$ on $X$ such that, if $X$ is oriented by $\omega \wedge \omega$ and $\partial X$ is given by the boundary orientation, there exists an orientation-preserving diffeomorphism $\varphi: Y \rightarrow \partial X$ such that $\left.\omega\right|_{d \varphi(\xi)} \neq 0$ at every point of $\partial X$. For example, the unit four-ball $B^{4} \subset \mathbb{C}^{2}$ endowed with the restriction of the standard Kähler form on $\mathbb{C}^{2}$ is a symplectic filling of $\left(S^{3}, \xi_{\mathrm{st}}\right)$, where the standard contact structure $\xi_{\mathrm{st}}$ on $S^{3}$ is, by definition, the distribution of complex lines tangent to $S^{3} \subset \mathbb{C}^{2}$.

The first classification result for symplectic fillings is due to Eliashberg [6], who proved that if $(X, \omega)$ is a symplectic filling of $\left(S^{3}, \xi_{\mathrm{st}}\right)$, then $X$ is diffeomorphic to a blowup of $B^{4}$. McDuff [19] extended Eliashberg's result to the lens spaces $L(p, 1)$ endowed with the contact structure $\bar{\xi}_{\text {st }}$ defined in the following paragraph. Ohta and Ono [23] determined the diffeomorphism types of the strong symplectic fillings of links of simple elliptic singularities endowed with their natural contact structures. In $[15,24]$ results on the intersection forms of symplectic fillings of finite quotients of $S^{3}$ were proved.

The distribution $\xi_{\text {st }}$ on $S^{3}$, being invariant under the natural action of $U(2)$, is a fortiori invariant under the induced action of the subgroup

$$
G_{p, q}=\left\{\left(\begin{array}{cc}
\zeta & 0 \\
0 & \zeta^{q}
\end{array}\right) \mid \zeta^{p}=1\right\} \subset U(2),
$$

Received by the editors October 11, 2005.

2000 Mathematics Subject Classification. Primary 57R17; Secondary 53D35.

(C) 2007 American Mathematical Society Reverts to public domain 28 years from publication 
where $p, q \in \mathbb{Z}$. It follows that when $p>q \geq 1$ and $p, q$ are coprime, $\xi_{\text {st }}$ descends to a contact structure $\bar{\xi}_{\text {st }}$ on the lens space $L(p, q)=S^{3} / G_{p, q}$.

Let $D_{p}$ denote the disk bundle over the two-sphere with Euler class $e\left(D_{p}\right)=p$. The contact three-manifold $\left(L(p, 1), \bar{\xi}_{\mathrm{st}}\right)$ admits a symplectic filling of the form $\left(D_{-p}, \omega\right)$ for every $p>1$. On the other hand, $\left(L(4,1), \bar{\xi}_{\text {st }}\right)$ also admits a symplectic filling of the form $\left(\mathbb{C P}^{2} \backslash \nu(C), \omega_{0}\right)$, where $\nu(C)$ is a strictly pseudo-concave neighborhood of a smooth conic $C \subset \mathbb{C P}^{2}$ and $\omega_{0}$ is the restriction of the standard Kähler form on $\mathbb{C P}^{2}$.

McDuff proved [19] that if $(X, \omega)$ is a symplectic filling of $\left(L(p, 1), \bar{\xi}_{\text {st }}\right)$, then $X$ is orientation preserving diffeomorphic to a smooth blowup of:

(a) $D_{-p}$ if $p \neq 4$,

(b) $D_{-4}$ or $\mathbb{C P}^{2} \backslash \nu(C)$ if $p=4$.

In this paper we obtain a complete classification up to diffeomorphism for the symplectic fillings of the contact three-manifolds $\left(L(p, q), \bar{\xi}_{\mathrm{st}}\right)$. In order to state our result we need to introduce some notation.

Let $p$ and $q$ be coprime integers such that

$$
p>q \geq 1, \quad \frac{p}{p-q}=b_{1}-\frac{1}{b_{2}-\frac{1}{\ddots \cdot-\frac{1}{b_{k}}}}, \quad b_{1}, \ldots, b_{k} \geq 2 .
$$

The integers $b_{i}$ are uniquely determined by the rational number $\frac{p}{p-q}$. Without the assumption $b_{i} \geq 2$, this uniqueness property fails. The standard symbol $\left[b_{1}, \ldots, b_{k}\right]$ will be used throughout the paper to denote a contined fraction as the one in (1.2).

Definition. A $k$-tuple of non-negative integers $\left(n_{1}, \ldots, n_{k}\right)$ is admissible if each of the denominators appearing in the continued fraction $\left[n_{1}, \ldots, n_{k}\right]$ is positive.

Let $\mathbf{n}=\left(n_{1}, \ldots, n_{k}\right)$ be an admissible $k$-tuple of non-negative integers such that

$$
\left[n_{1}, \ldots, n_{k}\right]=0 .
$$

Let $N(\mathbf{n})$ be the closed, oriented three-manifold given by surgery on $S^{3}$ along the framed link of Figure 1. It is easy to check that assumption (1.3) ensures the existence of an orientation preserving diffeomorphism

$$
\varphi: N(\mathbf{n}) \rightarrow S^{1} \times S^{2} .
$$

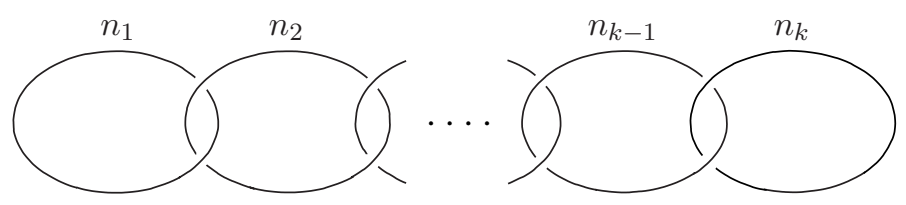

Figure 1. The manifold $N(\mathbf{n})$

Definition. Fix a diffeomorphism $\varphi$ as in (1.4), and let $\mathbf{L} \subset N(\mathbf{n})$ be the thick framed link drawn in Figure 2. Define $W_{p, q}(\mathbf{n})$ to be the smooth four-manifold with boundary obtained by attaching two-handles to $S^{1} \times D^{3}$ along the framed link

$$
\varphi(\mathbf{L}) \subset S^{1} \times S^{2} .
$$


Remark. The diffeomorphism type of $W_{p, q}(\mathbf{n})$ is independent of the choice of the diffeomorphism $\varphi$, because every self-diffeomorphism of $S^{1} \times S^{2}$ extends to $S^{1} \times$ $D^{3}[10]$.

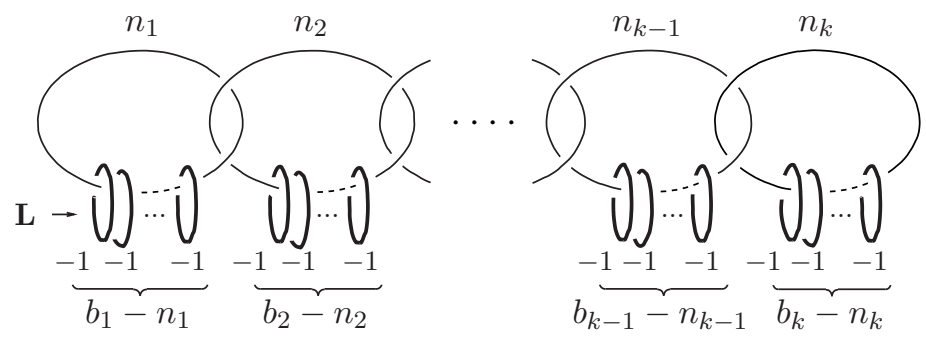

Figure 2. The framed link $\mathbf{L} \subset N(\mathbf{n})$

Definition. Let $\mathbf{Z}_{p, q} \subset \mathbb{Z}^{k}$ be the set of admissible $k$-tuples of non-negative integers $\left(n_{1}, \ldots, n_{k}\right)$ such that

$$
\left[n_{1}, \ldots, n_{k}\right]=0 \quad \text { and } \quad 0 \leq n_{i} \leq b_{i} \text { for } i=1, \ldots, k \text {. }
$$

It is easy to check (see Section 2) that the set $\mathbf{Z}_{p, q}$ admits the involution:

$$
\mathbf{n}=\left(n_{1}, \ldots, n_{k}\right) \mapsto \overline{\mathbf{n}}=\left(n_{k}, \ldots, n_{1}\right) .
$$

Given coprime integers $p>q \geq 1$, we denote by $\bar{q}$ the only integer satifsying

$$
p>\bar{q} \geq 1, \quad q \bar{q} \equiv 1 \bmod p .
$$

Theorem 1.1. Let $p>q \geq 1$ be coprime integers. Then,

(1) Let $(W, \omega)$ be a symplectic filling of the contact three-manifold $\left(L(p, q), \bar{\xi}_{\mathrm{st}}\right)$. Then, there exists $\mathbf{n} \in \mathbf{Z}_{p, q}$ such that $W$ is orientation preserving diffeomorphic to a smooth blowup of $W_{p, q}(\mathbf{n})$.

(2) For every $\mathbf{n} \in \mathbf{Z}_{p, q}$, the four-manifold $W_{p, q}(\mathbf{n})$ carries a symplectic form $\omega$ such that $\left(W_{p, q}(\mathbf{n}), \omega\right)$ is a symplectic filling of the contact three-manifold $\left(L(p, q), \bar{\xi}_{\mathrm{st}}\right)$. Moreover, there are no classes in $H_{2}\left(W_{p, q}(\mathbf{n}) ; \mathbb{Z}\right)$ with selfintersection equal to -1 .

(3) Let $\mathbf{n} \in \mathbf{Z}_{p, q}$ and $\mathbf{n}^{\prime} \in \mathbf{Z}_{p^{\prime}, q^{\prime}}$. Then, $W_{p, q}(\mathbf{n}) \# r \overline{\mathbb{C P}}^{2}$ is orientation preserving diffeomorphic to $W_{p^{\prime}, q^{\prime}}\left(\mathbf{n}^{\prime}\right) \# s \overline{\mathbb{C P}}^{2}$ if and only if:

(a) $\left(p^{\prime}, s\right)=(p, r)$ and $\left(q^{\prime}, \mathbf{n}^{\prime}\right)=(q, \mathbf{n})$, or

(b) $\left(p^{\prime}, s\right)=(p, r)$ and $\left(q^{\prime}, \mathbf{n}^{\prime}\right)=(\bar{q}, \overline{\mathbf{n}})$.

Theorem 1.1 gives a complete diffeomorphism classification of the symplectic fillings of $\left(L(p, q), \bar{\xi}_{\mathrm{st}}\right)$, extending the result of McDuff quoted above. ${ }^{1}$ For instance, as explained in [16], by Theorem 1.1 there are exactly two symplectic fillings of $\left(L\left(p^{2}, p-1\right), \bar{\xi}_{\mathrm{st}}\right)$ up to blowups and diffeomorphisms. One comes from the canonical resolution of the associated singularity, while the other is the rational homology ball used in the symplectic rational blowdown construction [8, 27].

\footnotetext{
${ }^{1}$ Portions (1) and (2) of Theorem 1.1 were announced in $[16,17]$.
} 
The following corollary gives three new results as applications of Theorem 1.1:

Corollary 1.2. Let $p>q \geq 1$ be coprime integers.

(a) Given a positive integer $n$, there exist infinitely many lens spaces $L(p, q)$ such that the contact three-manifold $\left(L(p, q), \bar{\xi}_{\mathrm{st}}\right)$ has more than $n$ symplectic fillings pairwise distinct up to homotopy equivalence and whose underlying four-manifolds are smoothly minimal. ${ }^{2}$

(b) There exist infinitely many lens spaces $L(p, q)$ such that $q>1$ and the contact three-manifold $\left(L(p, q), \bar{\xi}_{\mathrm{st}}\right)$ has only one symplectic filling up to blowups and diffeomorphisms.

(c) The contact three-manifold $\left(L(p, q), \bar{\xi}_{\mathrm{st}}\right)$ has a symplectic filling $(W, \omega)$ with $b_{2}(W)=0$ if and only if $(p, q)=\left(m^{2}, m h-1\right)$, for some $m$ and $h$ coprime natural numbers.

The statement of Theorem 1.1 - not the proof given here - is related to the deformation theory of complex two-dimensional cyclic quotient singularities. In fact, the contact three-manifold $\left(L(p, q), \bar{\xi}_{\text {st }}\right)$ can be viewed as the link of the singularity $\left(\mathbb{C}^{2} / G_{p, q}, 0\right)$ together with the natural contact structure given by the complex tangents. Every smoothing of $\left(\mathbb{C}^{2} / G_{p, q}, 0\right)$ determines Stein fillings $F$ of $\left(L(p, q), \bar{\xi}_{0}\right)$, and Theorem 1.1 implies that any such $F$ must be diffeomorphic to $W_{p, q}(\mathbf{n})$ for some $\mathbf{n} \in \mathbf{Z}_{p, q}$. It seems likely that a converse to this fact should also hold, because every irreducible component of the reduced base space $S_{\text {red }}$ of the versal deformation of $\left(\mathbb{C}^{2} / G_{p . q}, 0\right)$ gives a smoothing of the singularity, and Stevens [26] proved the existence of a one-to-one correspondence between $\mathbf{Z}_{p, q}$ and the set $\mathcal{S}_{p, q}$ of irreducible components of $S_{\text {red }}$ (see also [3]). In light of the results obtained in this paper, we propose the following:

Conjecture. Let $F(\mathbf{n})$ be a Stein filling of $\left(L(p, q), \bar{\xi}_{\mathrm{st}}\right)$ determined by the smoothing of $\left(\mathbb{C}^{2} / G_{p . q}, 0\right)$ corresponding to $\mathbf{n} \in \mathbf{Z}_{p, q}$ under the Stevens correspondence [26]. Then, $F(\mathbf{n})$ is diffeomorphic to $W_{p, q}(\mathbf{n})$.

There is evidence supporting this conjecture. In fact, in this paper we prove that each $W_{p, q}(\mathbf{n})$ carries Stein structures (Corollary 5.2). Moreover, the smoothing corresponding to $(1,2, \ldots, 2,1) \in \mathbf{Z}_{p, q}$ is known to be isomorphic to the canonical resolution $X_{p, q}$ of the singularity, and it is not hard to verify that if $\mathbf{n}=(1,2, \ldots, 2,1)$, then $W_{p, q}(\mathbf{n})$ is indeed diffeomorphic to a regular neighborhood of the exceptional divisor in $X_{p, q}$. Finally, by $[28,5.9 .1]$, a singularity $\left(\mathbb{C}^{2} / G_{p . q}, 0\right)$ has a smoothing with $b_{2}=0$ if and only if $(p, q)=\left(m^{2}, m h-1\right)$, with $m$ and $h$ coprime, in agreement with Corollary 1.2(c).

The paper is organized as follows. In Section 2 we prove Corollary 1.2 assuming Theorem 1.1. In Section 3 we show that every symplectic filling $(W, \omega)$ of $\left(L(p, q), \bar{\xi}_{\text {st }}\right)$ can be compactified to a rational symplectic four-manifold $X$ so that $X \backslash W$ is a neighborhood of an immersed symplectic surface $\Gamma \subset X$ of a special kind. In Section 4 we prove Theorem 1.1(1). In Section 5 we construct Stein structures on the smooth four-manifolds $W_{p, q}(\mathbf{n})$. In Section 6 we prove Theorem 1.1(2). In Section 7 we complete the proof of Theorem 1.1.

\footnotetext{
${ }^{2}$ A smooth, oriented four-manifold $X$ is smoothly minimal if the interior of $X$ contains no smoothly embedded two-sphere of self-intersection -1 .
} 


\section{The Proof of Corollary 1.2}

Definition 2.1. Given a $k$-tuple of positive integers

$$
\left(n_{1}, \ldots, n_{s-1}, n_{s}, n_{s-1}, \ldots, n_{k}\right)
$$

with $n_{s}=1$, we say that the $(k-1)$-tuple

$$
\left(n_{1}, \ldots, n_{s-1}-1, n_{s+1}-1, \ldots, n_{k}\right)
$$

is obtained by a blowdown at $n_{s}$ (with the obvious meaning of the notation when $s=1$ or $s=k$ ). The reverse process is a blowup. A blowdown at $n_{s}$ is strict if $s>1$. The reverse process is a strict blowup.

It is shown in [22, Appendix] that a $k$-tuple of positive integers $\left(n_{1}, \ldots, n_{k}\right)$ is admissible $^{3}$ if and only if the symmetric matrix

$$
\left(\begin{array}{ccccc}
n_{1} & -1 & & & \\
-1 & n_{2} & -1 & & \\
& -1 & n_{3} & & \\
& & & \ddots & -1 \\
& & & -1 & -n_{k}
\end{array}\right)
$$

is positive semi-definite of rank $\geq k-1$. It immediately follows from this fact that if $\left(n_{1}, \ldots, n_{k}\right)$ is admissible, then $\left(n_{k}, \ldots, n_{1}\right)$ is admissible,

$$
\left(n_{i}, n_{i+1}, \ldots, n_{j-1}, n_{j}\right)
$$

is admissible for every $1 \leq i \leq j \leq k$, and blowing up and blowing down preserve admissibility.

Lemma 2.2. Let $\left(n_{1}, \ldots, n_{k}\right)$ be a $k$-tuple of positive integers. Then, the following conditions are equivalent:

- $\left(n_{1}, \ldots, n_{k}\right)$ is admissible and satisfies $\left[n_{1}, \ldots, n_{k}\right]=0$;

- $\left(n_{1}, \ldots, n_{k}\right)$ is obtained from (0) by a sequence of strict blowups.

Proof. Clearly, a $k$-tuple of positive integers is obtained from (0) via a sequence of strict blowups if and only if it is obtained from $(1,1)$ via such a sequence. Moreover, both the property of being admissible and that of having a vanishing associated continued fraction are preserved under blowup. Therefore, since $(1,1)$ is admissible and $[1,1]=0$, any $k$-tuple of positive integers obtained from (0) by a sequence of strict blowups is admissible and has a vanishing associated continued fraction.

Conversely, let $\left(n_{1}, \ldots, n_{k}\right)$ be an admissible $k$-tuple of positive integers with $\left[n_{1}, \ldots, n_{k}\right]=0$. Then, we must have $k \geq 2$. We shall argue by induction that $\left(n_{1}, \ldots, n_{k}\right)$ is obtained from $(0)$ by a sequence of strict blowups. For $k=2$ the statement is obvious, so suppose that $k>2$ and the statement corresponding to $k-1$ holds true. An easy induction argument shows that if $n_{i} \geq 2$ for every $i=1, \ldots, k$, then $\left[n_{1}, \ldots, n_{k}\right]>1$. Thus, if $\left[n_{1}, \ldots, n_{k}\right]=0$, then necessarily $n_{i}=1$ for some $i \in\{1, \ldots, k\}$. We conclude that $\left(n_{1}, \ldots, n_{k}\right)$ is obtained from the admissible $(k-1)$-tuple

$$
\left(n_{1}, \ldots, n_{i-1}-1, n_{i+1}-1, \ldots, n_{k}\right)
$$

\footnotetext{
${ }^{3}$ The definition of admissibility given in [22, Appendix] is easily seen to be equivalent to the one given in Section 1.
} 
by a blowup (which is strict if and only if $i>1$ ). By the induction hypothesis,

$$
\left(n_{1}, \ldots, n_{i-1}-1, n_{i+1}-1, \ldots, n_{k}\right)
$$

is obtained from (0) by a sequence of strict blowups, hence $n_{j}=1$ for some $j>$ 1. Therefore, the $k$-tuple $\left(n_{1}, \ldots, n_{k}\right)$ is obtained by a strict blowup from the admissible $(k-1)$-tuple

$$
\left(n_{1}, \ldots, n_{j-1}-1, n_{j+1}-1, \ldots, n_{k}\right),
$$

and the conclusion follows by induction.

Lemma 2.3. Let $p>q \geq 1$ be coprime integers, and suppose that

$$
\frac{p}{q}=\left[a_{1}, \ldots, a_{h}\right], \quad \text { with } a_{1}, \ldots, a_{h} \geq 5 .
$$

Then,

$$
\mathbf{Z}_{p, q}=\{(1,2, \ldots, 2,1)\}
$$

Proof. Let

$$
\frac{p}{p-q}=\left[b_{1}, \ldots, b_{k}\right], \quad b_{1}, \ldots, b_{k} \geq 2 .
$$

Using Riemenschneider's point diagram [25], one can easily check that the assumption on the $a_{i}$ 's implies the following three conditions:

- $k \geq 4$,

- $b_{1}, \ldots, b_{k} \leq 3$,

- if $b_{i}=b_{j}=3$, then either $3<i=j<k-2$ or $|i-j| \geq 3$.

Therefore, if $\left(n_{1}, \ldots, n_{k}\right) \in \mathbf{Z}_{p, q}$ we have

(1) $k \geq 4$,

(2) $n_{i} \leq 3$ for every $i=1, \ldots, k$,

(3) if $n_{i}=n_{j}=3$, then either $3<i=j<k-2$ or $|i-j| \geq 3$.

We shall argue by induction on $k \geq 4$ that if $\left(n_{1}, \ldots, n_{k}\right)$ is an admissible sequence of non-negative integers such that $\left[n_{1}, \ldots, n_{k}\right]=0$ and such that (1), (2) and (3) above hold, then

$$
\left(n_{1}, \ldots, n_{k}\right)=(1,2, \ldots, 2,1) .
$$

If $k=4$ one immediately sees that, by Lemma 2.2 and Assumption (3),

$$
\left(n_{1}, n_{2}, n_{3}, n_{4}\right)=(1,2,2,1) .
$$

Now suppose $k>4$. By Lemma 2.2, $n_{i}>0$ for every $i=1, \ldots, k$ and $n_{j}=1$ for some index $j>1$. We claim that $j=k$. In fact, suppose $j<k$. If $j=2$ or $j=k-1$, then Assumption (3) implies $n_{j-1}=n_{j+1}=2$. By Lemma 2.2 this is impossible, because two blowdowns would give a string of length bigger than 1 containing 0 . Therefore we have $2<j<k-1$. Blowing down once yields the sequence

$$
\left(n_{1}, \ldots, n_{j-1}-1, n_{j+1}-1, \ldots, n_{k}\right),
$$

which still satisfies the three assumptions. Therefore, by induction

$$
\left(n_{1}, \ldots, n_{j-1}-1, n_{j+1}-1, \ldots, n_{k}\right)=(1,2, \ldots, 2,1) .
$$

But then $n_{j-1}=n_{j+1}=3$, which goes against Assumption (3). We conclude that $j=k$. Blowing down once yields the sequence

$$
\left(n_{1}, \ldots, n_{k-1}-1\right),
$$


which satisfies the assumptions if and only if $n_{k-3}<3$. Notice that if $n_{k-3}<3$, we can apply induction and reach the conclusion. Blowing down two more times yields the sequence

$$
\left(n_{1}, \ldots, n_{k-3}-1\right),
$$

which satisfies the assumptions, so by induction we get $n_{k-3}=2$.

We need the following elementary facts about continued fractions (see e.g. [22, Appendix] for the proofs):

- Let $p>q \geq 1$ be coprime integers, and suppose that

$$
\frac{p}{q}=\left[a_{1}, a_{2}, \ldots, a_{h}\right], \quad a_{1}, \ldots, a_{h} \geq 2 .
$$

Then,

where $p>\bar{q} \geq 1$ and $q \bar{q} \equiv 1 \bmod p$.

$$
\left[a_{h}, a_{h-1}, \ldots, a_{1}\right]=\frac{p}{\bar{q}}
$$

- If $\left(n_{1}, \ldots, n_{k}\right)$ is an admissible $k$-tuple of positive integers, then

$$
\left[n_{1}, n_{2}, \ldots, n_{k}\right]=0
$$

if and only if

$$
\left[n_{k}, n_{k-1} \ldots, n_{1}\right]=0 .
$$

Lemma 2.4. Let $\left(n_{1}, \ldots, n_{k}\right)$ be an admissible $k$-tuple of positive integers such that

$$
\left[n_{1}, n_{2}, \ldots, n_{k}\right]=0, \quad k \geq 3 .
$$

Suppose that there is exactly one index $j \in\{1, \ldots, k\}$ such that $n_{j}=1$. Then, there are coprime integers $m$ and $n$ such that

$$
\left[n_{1}, n_{2}, \ldots, n_{j-1}, 2, n_{j+1}, \ldots, n_{k}\right]=\frac{m^{2}}{m n+1} .
$$

Proof. We argue by induction on $k \geq 3$. For $k=3$, the assumptions and Lemma 2.2 imply

$$
\left(n_{1}, n_{2}, n_{3}\right)=(2,1,2)
$$

and

$$
[2,2,2]=\frac{4}{3}
$$

is of the stated form. Now suppose $k>3$. Since, by Lemma $2.2,\left(n_{1}, \ldots, n_{k}\right)$ must be obtained from $(2,1,2)$ by a sequence of strict blowups, we have

$$
n_{1}=2, n_{k}>2, \quad \text { or } n_{1}>2, n_{k}=2 .
$$

Observe that

if and only if

$$
\left[n_{1}, n_{2}, \ldots, n_{j-1}, 2, n_{j+1}, \ldots, n_{k}\right]=\frac{m^{2}}{m n+1}
$$

$$
\left[n_{k}, n_{k-1}, \ldots, n_{j+1}, 2, n_{j-1}, \ldots, n_{1}\right]=\frac{m^{2}}{m(m-n)+1} .
$$

Therefore, without loss of generality we may assume

$$
n_{1}=2 \text { and } n_{k}>2 .
$$

Under these assumptions we claim that

$$
\left[n_{2}, \ldots, n_{k}-1\right]=0 .
$$


In order to prove the claim, we argue by induction on $k \geq 4$. If $k=4$ we must have

$$
\left(n_{1}, n_{2}, n_{3}, n_{4}\right)=(2,2,1,3),
$$

and the claim is clear. If $k>4$, observe that, since by Lemma $2.2,\left(n_{1}, \ldots, n_{k}\right)$ is a blowup of $(2,2,1,3)$, we must have $j>2$. Then, blowing down once yields the string

$$
\left(n_{1}=2, \ldots, n_{j-1}-1, n_{j+1}-1, \ldots, n_{k}\right),
$$

to which we may apply induction to conclude

$$
\left[n_{2}, \ldots, n_{j-1}-1, n_{j+1}-1, \ldots, n_{k}-1\right]=0
$$

if $j<k-1$, and

$$
\left[n_{2}, \ldots, n_{k-2}-1, n_{k}-2\right]=0
$$

if $j=k-1$. Blowing up again proves the claim (2.1).

Now induction applied to (2.1) gives

$$
\left[n_{2}, \ldots, n_{j-1}, 2, n_{j+1}, \ldots, n_{k}-1\right]=\frac{n^{2}}{n h+1},
$$

with $n, h$ coprime integers. Since

$$
(1+n h)(1-n h) \equiv 1 \bmod n^{2},
$$

we have

$$
\left[n_{k}-1, \ldots, n_{j+1}, 2, n_{j-1}, \ldots, n_{2}\right]=\frac{n^{2}}{n(n-h)+1}
$$

therefore

$$
\left[n_{k}, \ldots, n_{j+1}, 2, n_{j-1}, \ldots, n_{2}\right]=\frac{n^{2}}{n(n-h)+1}+1=\frac{2 n^{2}-n h+1}{n(n-h)+1} .
$$

Thus, since

$$
\left(n^{2}-n h+1\right)\left(2 n h-h^{2}+2\right)=\left(2 n^{2}-n h+1\right)\left(n h-h^{2}+1\right)+1,
$$

we conclude

$$
\left[n_{1}=2, n_{2}, \ldots, n_{j-1}, 2, n_{j+1}, \ldots, n_{k}\right]=2-\frac{2(n h+1)-h^{2}}{2 n^{2}-n h+1}=\frac{m^{2}}{m n+1},
$$

where $m=2 n-h$.

Proof of Corollary 1.2. (a) Let $p>q \geq 1$ be coprime and such that

$$
\frac{p}{p-q}=\left[b_{1}, \ldots, b_{k}\right], \quad b_{1}, \ldots, b_{k} \geq 2,
$$

with $k \geq 4, b_{2}, \ldots, b_{k-2} \geq 3$ and $b_{k} \geq k-2$. Let $r \in \mathbb{Z}, 0 \leq r \leq k-4$. Then,

$$
\mathbf{n}_{r}=(1, \overbrace{2, \ldots, 2}^{r}, 3, \overbrace{2, \ldots, 2}^{k-4-r}, 1, k-2-r) \in \mathbf{Z}_{p, q} .
$$

One can easily check that

$$
\chi\left(W_{p, q}\left(\mathbf{n}_{r}\right)\right)=5+\sum_{i=1}^{k}\left(b_{i}-3\right)+r .
$$

Then, Equation (2.2) and Theorem 1.1 imply that $\left(L(p, q), \bar{\xi}_{\text {st }}\right)$ admits at least $k-3$ smoothly minimal symplectic fillings up to homotopy equivalence. 
(b) If

$$
\frac{p}{q}=\left[a_{1}, \ldots, a_{h}\right], \quad a_{1}, \ldots, a_{h} \geq 5,
$$

then by Lemma 2.3

$$
\mathbf{Z}_{p, q}=\{(1,2, \ldots, 2,1)\} .
$$

The conclusion follows by Theorem 1.1.

(c) Suppose that

$$
\frac{p}{p-q}=\left[b_{1}, \ldots, b_{k}\right]
$$

It is easy to check that the attaching circle of each two-handle of $W_{p, q}(\mathbf{n})$ is homologically non-trivial in $S^{1} \times S^{2}$. Therefore, $b_{2}\left(W_{p, q}(\mathbf{n})\right)=0$ if and only if there is exactly one index $j \in\{1, \ldots, k\}$ such that $n_{j}=1$, and

$$
\left(b_{1}, \ldots, b_{k}\right)=\left(n_{1}, n_{2}, \ldots, n_{j-1}, 2, n_{j+1}, \ldots, n_{k}\right) .
$$

Then, by Lemma 2.4,

$$
\frac{p}{p-q}=\frac{m^{2}}{m n+1}
$$

with $m$ and $n$ coprime. Therefore $q=m h-1$, with $h=m-n$.

\section{CompaCtifications OF SYMPleCtic FILlings}

The purpose of this section is to prove Theorem 3.2 below. In order to state the theorem, we need a definition.

Definition 3.1. Let $(X, \omega)$ be a symplectic four-manifold. A symplectic string in $X$ is an immersed symplectic surface

$$
\Gamma=C_{0} \cup C_{1} \cup \cdots \cup C_{k} \subset X,
$$

where:

- $C_{i}$ is a connected, embedded symplectic sphere for $i=0, \ldots, k$;

- $C_{i}$ and $C_{i+1}$ intersect transversely and positively at a single point, for $i=0, \ldots, k-1$

- $C_{i} \cap C_{j}=\emptyset$ if $|i-j|>1$, for $i, j=0, \ldots, k$.

We say that $\Gamma$ as above is of type $\left(m_{0}, \ldots, m_{k}\right)$ if, furthermore,

- $C_{i} \cdot C_{i}=m_{i}$ for $i=0, \ldots, k$.

Theorem 3.2. Let $p>q \geq 1$ be coprime integers, and suppose that

$$
\frac{p}{p-q}=\left[b_{1}, \ldots, b_{k}\right], \quad b_{1}, \ldots, b_{k} \geq 2 .
$$

Let $(W, \omega)$ be a symplectic filling of $\left(L(p, q), \bar{\xi}_{\text {st }}\right)$. Then, for some integer $M \geq 0, W$ is orientation preserving diffeomorphic to the complement of a regular neighborhood of a symplectic string

$$
\Gamma=C_{0} \cup C_{1} \cup \cdots \cup C_{k} \subset \mathbb{C P}^{2} \# M \overline{\mathbb{C P}}^{2}
$$

of type $\left(1,1-b_{1},-b_{2}, \ldots,-b_{k}\right)$, where $\mathbb{C P}^{2} \# M \overline{\mathbb{C P}}^{2}$ is endowed with a symplectic blowup $\omega_{M}$ of the standard Kähler form on $\mathbb{C P}^{2}$, and $C_{0}$ is a complex line in $\mathbb{C P}^{2}$. 
Theorem 3.2 will be used in Section 4 . We start with an auxiliary construction of a suitable symplectic form on $(0, \infty) \times L(p, q)$.

The function

$$
\begin{aligned}
& \rho: \quad \mathbb{C}^{2} \longrightarrow(0,+\infty), \\
& \left(z_{1}, z_{2}\right) \mapsto\left|z_{1}\right|^{2}+\left|z_{2}\right|^{2}
\end{aligned}
$$

is a Kähler potential for the standard symplectic form $\omega_{0}$, i.e.

$$
\omega_{0}=\frac{i}{2} \sum_{k=1}^{2} d z_{k} \wedge d \bar{z}_{k}=\frac{i}{2} \partial \bar{\partial} \rho
$$

Let $J_{0}$ be the standard complex structure on $\mathbb{C}^{2}$, and consider the one-form

$$
\sigma_{0}=-\frac{1}{4} d \rho \circ J_{0}=-\frac{i}{4}(\partial \rho-\bar{\partial} \rho)=-\frac{i}{4} \sum_{k=1}^{2}\left(\bar{z}_{k} d z_{k}-z_{k} d \bar{z}_{k}\right) .
$$

Let $i: S^{3}=\rho^{-1}(1) \hookrightarrow \mathbb{C}^{2}$ be the inclusion map, and define $\alpha_{0}=i^{*} \sigma_{0} \in \Omega^{1}\left(S^{3}\right)$. Since the standard contact structure $\xi_{\text {st }}$ on $S^{3}$ is given by complex tangent lines, we have $\xi_{\text {st }}=\left\{\alpha_{0}=0\right\} \subset T S^{3}$. Define $\pi: \mathbb{C}^{2} \backslash\{(0,0)\} \rightarrow S^{3}$ by setting:

$$
\pi(\mathbf{z})=\frac{\mathbf{z}}{\rho(\mathbf{z})^{\frac{1}{2}}} .
$$

The pair $(\rho, \pi)$ induces an orientation preserving diffeomorphism:

$$
(\rho, \pi): \mathbb{C}^{2} \backslash\{(0,0)\} \rightarrow(0,+\infty) \times S^{3} .
$$

The diffeomorphism $(\rho, \pi)$ sends the standard symplectic form $\omega_{0}$ to the form $d\left(t \alpha_{0}\right)$, where $t$ is the coordinate on the first factor. To see this, notice that

$$
(i \circ \pi)^{*} \sigma_{0}=-\frac{i}{4} \sum_{k=1}^{2}\left(\bar{z}_{k} d\left(\frac{z_{k}}{\rho(\mathbf{z})^{\frac{1}{2}}}\right)-z_{k} d\left(\frac{\bar{z}_{k}}{\rho(\mathbf{z})^{\frac{1}{2}}}\right)\right)=\frac{1}{\rho} \sigma_{0},
$$

and therefore:

$$
(\rho, \pi)^{*} d\left(t \alpha_{0}\right)=d\left(\rho \pi^{*} i^{*} \sigma_{0}\right)=d \sigma_{0}=-\frac{i}{4}(\bar{\partial} \partial \rho-\partial \bar{\partial} \rho)=\frac{i}{2} \partial \bar{\partial} \rho .
$$

Since $U(2)$ acts on $\mathbb{C}^{2}$ via norm-preserving complex linear transformations, $\sigma_{0}$ and $\omega_{0}$ are clearly $U(2)$-invariant, while $\alpha_{0}$ is invariant under the naturally induced action on $S^{3}$. Moreover, $(\rho, \pi)$ is $U(2)$-equivariant in an obvious sense, so we have:

- a symplectic form $\bar{\omega}_{0}$ induced by $\omega_{0}$ on $\mathbb{C}^{2} \backslash\{(0,0)\} / G_{p, q}$, where $G_{p, q}$ is the subgroup of $U(2)$ defined by (1.1),

- a contact form $\bar{\alpha}_{0}$ induced by $\alpha_{0}$ on $L(p, q)=S^{3} / G_{p, q}$, and

- a symplectomorphism

$$
\left(\mathbb{C}^{2} \backslash\{(0,0)\} / G_{p, q}, \bar{\omega}_{0}\right) \cong\left((0,+\infty) \times L(p, q), d\left(t \bar{\alpha}_{0}\right)\right) .
$$

With the notation of Section 1 , we have $\bar{\xi}_{\text {st }}=\left\{\bar{\alpha}_{0}=0\right\}$. The action

$$
\begin{aligned}
S^{1} \times S^{3} & \longrightarrow S^{3}, \\
\left(e^{i \theta}, \mathbf{z}\right) & \longmapsto e^{i \theta} \mathbf{z}
\end{aligned}
$$

commutes with the action of $G_{p, q}$, so it induces a fixed-point free $S^{1}$-action:

$$
S^{1} \times L(p, q) \longrightarrow L(p, q) .
$$


There is also an obviously induced $S^{1}$-action on $(0,+\infty) \times L(p, q)$. Since $\alpha_{0}$ is $U(2)$-invariant, the symplectic form $d\left(t \bar{\alpha}_{0}\right)$ is invariant under this $S^{1}$-action.

The hypersurfaces

$$
\{t\} \times L(p, q) \subset(0,+\infty) \times L(p, q)
$$

satisfy an important property with respect to the $S^{1}$-invariant symplectic form $d\left(t \bar{\alpha}_{0}\right)$. We shall establish this property after recalling its general context.

Let $(X, \omega)$ be a symplectic four-manifold and $Y \subset X$ a separating hypersurface endowed with a fixed-point free $S^{1}$-action. Following [21], we say that $Y$ is $\omega$ compatible if the orbits of the $S^{1}$-action lie in the null directions of $\left.\omega\right|_{Y}$. In general, we say that an embedding $j: Y \hookrightarrow X$ is $\omega$-compatible if $j(Y) \subset X$ is a separating $\omega$-compatible hypersurface.

An $\omega$-compatible hypersurface $Y$ has a canonical co-orientation. In fact, if $N \in T_{p} Y, p \in Y$, is the vector field generating the $S^{1}$-action on $Y$ then, for every vector $V \in T_{p} X$ transverse to $Y, \omega(N, V) \neq 0$, for otherwise $\omega$ would be degenerate on $T_{p} X$. Thus,

where

$$
T_{p} X \backslash T_{p} Y=T_{p}^{+} X \cup T_{p}^{-} X
$$

$$
T_{p}^{+} X=\left\{V \in T_{p} X \mid \omega(N, V)>0\right\}, \quad T_{p}^{-} X=\left\{V \in T_{p} X \mid \omega(N, V)<0\right\} .
$$

This allows one to distinguish the two components of $X \backslash Y$ by setting $X^{+}$to be the component with inward pointing normal vector $V \in T_{p}^{+} X$, for all $p \in Y$, and $X^{-}$to be remaining component. Thus, we have:

$$
X=X^{-} \cup_{Y} X^{+} \text {. }
$$

The importance of the $\omega$-compatibility condition is due to the fact that symplectic four-manifolds can be glued along $\omega$-compatible hypersurfaces. More precisely, J. McCarthy and J. Wolfson proved the following result:

Theorem 3.3 ([21], Theorem 4.2). Let $Y$ be a closed, oriented three-manifold with a fixed-point free $S^{1}$-action. Let $\left(X_{i}, \omega_{i}\right), i=1,2$, be symplectic four-manifolds and let $j_{i}: Y \rightarrow X_{i}, i=1,2$, be $\omega$-compatible embeddings. Then, there is a symplectic structure $\omega$ on a smooth four-manifold

$$
X=X_{1}^{-} \cup_{Y} X_{2}^{+}
$$

obtained by gluing $X_{1}^{-}$to $X_{2}^{+}$along $Y$. Moreover, there are neighborhoods $\nu_{i}\left(j_{i}(Y)\right)$ of $Y$ in $X_{i}, i=1,2$, such that

$$
\left.\omega\right|_{X_{2}^{+} \backslash \nu_{2}(Y)}=\omega_{2} \quad \text { and }\left.\quad \omega\right|_{X_{1}^{-} \backslash \nu_{1}(Y)}=c \omega_{1}
$$

for some constant $c>0$.

The idea of the proof of Theorem 3.2 is to show that any symplectic filling $(W, \omega)$ of $\left(L(p, q), \bar{\xi}_{\text {st }}\right)$ can be compactified in a suitable way. In order to do that, we first need to establish the following:

Proposition 3.4. Let $(W, \omega)$ be a symplectic filling of $\left(L(p, q), \bar{\xi}_{\mathrm{st}}\right)$. Let $(X, \eta)$ be a symplectic four-manifold and $j: L(p, q) \rightarrow X$ an embedding which is $\eta$-compatible with respect to the $S^{1}$-action (3.5). Then, there is a symplectic form $\bar{\omega}$ on a smooth four-manifold

$$
Z=W \cup_{\partial W=\partial X^{-}} X^{-}
$$

obtained by gluing $X^{-}$to $W$ along their boundaries. 
Proposition 3.4 will be proved after the next two lemmas.

Lemma 3.5. For every $t \in(0,+\infty)$, the hypersurface

$$
\{t\} \times L(p, q) \subset(0,+\infty) \times L(p, q)
$$

is $S^{1}$-invariant and $d\left(t \bar{\alpha}_{0}\right)$-compatible with respect to the action (3.5). Moreover,

$$
\begin{aligned}
& {[(0,+\infty) \times L(p, q)]^{+}=(0, t] \times L(p, q),} \\
& {[(0,+\infty) \times L(p, q)]^{-}=[t,+\infty) \times L(p, q) .}
\end{aligned}
$$

Proof. The hypersurface $\{t\} \times L(p, q)$ is clearly $S^{1}$-invariant for every $t \in(0,+\infty)$. In order to see that it is also $d\left(t \bar{\alpha}_{0}\right)$-compatible, we first show that the $S^{1}$-invariant hypersurface

$$
\rho^{-1}(t) \subset \mathbb{C}^{2} \backslash\{(0,0)\}
$$

is $\omega_{0}$-compatible. Given $\mathbf{z} \in \rho^{-1}(t)$, we have

$$
\left.\frac{d}{d \theta}\right|_{\theta=0}\left(e^{i \theta} \mathbf{z}\right)=i \mathbf{z} .
$$

Equivalently, the vector field

$$
N=J_{0}\left(z_{1} \frac{\partial}{\partial z_{1}}+z_{2} \frac{\partial}{\partial z_{2}}\right)
$$

generates the $S^{1}$-action. Since $\sigma_{0}$ is invariant,

$$
0=L_{N} \sigma_{0}=d i_{N} \sigma_{0}+i_{N} d \sigma_{0},
$$

and since $d \rho=\sum_{k} \bar{z}_{k} d z_{k}+z_{k} d \bar{z}_{k}$, we have

$$
i_{N} \sigma_{0}=-\frac{1}{4} d \rho\left(J_{0}(N)\right)=\frac{\rho}{4} .
$$

Therefore, by (3.7) we have

$$
\left.i_{N} d \sigma_{0}\right|_{\rho^{-1}(t)}=\left.i_{N} \omega_{0}\right|_{\rho^{-1}(t)}=0 .
$$

Equation (3.8) says that

$$
\rho^{-1}(t) \subset \mathbb{C}^{2} \backslash\{(0,0)\}
$$

is $\omega_{0}$-compatible. Therefore, by $U(2)$-invariance and (3.2) the hypersurface

$$
\{t\} \times L(p, q) \subset(0,+\infty) \times L(p, q)
$$

is $d\left(t \bar{\alpha}_{0}\right)$-compatible.

In order to prove the second part of the statement, by $U(2)$-invariance it is enough to check that, with respect to the $S^{1}$-action (3.3) and the $d\left(t \alpha_{0}\right)$-compatible hypersurface

$$
\{t\} \times S^{3} \subset(0,+\infty) \times S^{3},
$$

we have

$$
\left[(0,+\infty) \times S^{3}\right]^{+}=(0, t] \times S^{3}, \quad\left[(0,+\infty) \times S^{3}\right]^{-}=[t,+\infty) \times S^{3} .
$$

In fact, the vector field

$$
V=z_{1} \frac{\partial}{\partial z_{1}}+z_{2} \frac{\partial}{\partial z_{2}}
$$

is transverse to every hypersurface $\{t\} \times S^{3}$ and points inwardly with respect to

$$
[t,+\infty) \times S^{3} .
$$


Since $N=J_{0} V$, we have

$$
\omega_{0}(N, V)=\omega_{0}\left(J_{0} V, V\right)=-\omega_{0}\left(V, J_{0} V\right)<0 .
$$

This concludes the proof.

Lemma 3.6. Let $(W, \omega)$ be a symplectic filling of $\left(L(p, q), \bar{\xi}_{\mathrm{st}}\right)$. Then, there exists a symplectic four-manifold $(\widetilde{W}, \widetilde{\omega})$ with one end $\mathcal{E}$ such that:

(a) $\left(\mathcal{E},\left.\widetilde{\omega}\right|_{\mathcal{E}}\right)$ is symplectomorphic to

$$
\left((D,+\infty) \times L(p, q), d\left(t \bar{\alpha}_{0}\right)\right)
$$

for some constant $D>0$;

(b) $\widetilde{W} \backslash \mathcal{E}$ is orientation preserving diffeomorphic to $W$.

Proof. Choose an open collar $C \subset W$ around $\partial W$, and let

$$
\varphi: C \backslash \partial W \cong(0,+\infty) \times L(p, q)
$$

be an orientation preserving diffeomorphism such that, if we keep denoting by $\omega$ the push-forward of $\omega$ to

$$
(0,+\infty) \times L(p, q)
$$

and we identify $\{1\} \times L(p, q)$ with $L(p, q)$, we have $\left.\omega\right|_{\bar{\xi}_{\text {st }}} \neq 0$. In fact, we claim that there exists a contactomorphism

$$
\bar{c}:\left(L(p, q), \bar{\xi}_{\mathrm{st}}\right) \rightarrow\left(L(p, q), \bar{\xi}_{\mathrm{st}}\right)
$$

which reverses the orientation of $\bar{\xi}_{\text {st }}$. Consequently, we can choose the identification of $\{1\} \times L(p, q)$ with $L(p, q)$ so that $\left.\omega\right|_{\bar{\xi}_{\text {st }}}>0$.

In order to prove the claim, recall that the standard contact structure $\xi_{\text {st }}$ on $S^{3}$ has a natural orientation induced by the standard complex structure on $\mathbb{C}^{2}$. The orientation on $\xi_{\text {st }}$ induces an orientation on $\bar{\xi}_{\text {st }}$. Moreover, the map $\mathbb{C}^{2} \rightarrow \mathbb{C}^{2}$ given by complex conjugation induces a contactomorphism

$$
c:\left(S^{3}, \xi_{\mathrm{st}}\right) \rightarrow\left(S^{3}, \xi_{\mathrm{st}}\right)
$$

which reverses the orientation of $\xi_{\text {st }}$. Since $c \circ A=A^{-1} \circ c$ for every element $A$ of the group $G_{p, q}$ defined in (1.1), $c$ induces the contactomorphism $\bar{c}$.

Since $L(p, q)$ has no non-trivial real two-cohomology, $\omega$ is exact. Eliashberg [5, Proposition 3.1] proved that in this situation there exist a contact form $\alpha \in$ $\Omega^{1}(L(p, q))$ defining $\bar{\xi}_{\text {st }}$, a constant $C>1$ and a symplectic form $\Omega$ on

$$
(0,+\infty) \times L(p, q)
$$

which coincides with $d(t \alpha)$ on

$$
[C,+\infty) \times L(p, q)
$$

and is equivalent to $\omega$ near $\{1\} \times L(p, q)$.

Eliashberg's argument is the following. Since $\left.\omega\right|_{\bar{\xi}_{\text {st }}}>0$, we have

$$
\left.\omega\right|_{\bar{\xi}_{\mathrm{st}}}=\left.h d \bar{\alpha}_{0}\right|_{\bar{\xi}_{\mathrm{st}}}
$$

for some smooth function $h: L(p, q) \rightarrow(0,+\infty)$. Let $\alpha=h \bar{\alpha}_{0}$. The restriction of $\omega$ to $L(p, q)$ is of the form $d \alpha+d \beta$ for some $\beta \in \Omega^{1}(L(p, q))$, and it follows from (3.9) that $d(t \alpha)+d \beta$ is a symplectic form near $\{1\} \times L(p, q)$. By the Symplectic Tubular Neighborhood Theorem, there exists a self-diffeomorphism $\psi$ of a tubular 
neighborhood of $\{1\} \times L(p, q)$ which is the identity restricted to $\{1\} \times L(p, q)$ and which sends $\omega$ to $d(t \alpha)+d \beta$. Let

$$
g:(0,+\infty) \rightarrow[0,1]
$$

be a smooth function which is identically 1 on $(0,1]$ and vanishes on $[C,+\infty)$ for some constant $C>1$. Then, if $\left|g^{\prime}(t)\right|$ is sufficiently small the two-form

$$
\Omega=d(t \alpha)+d(g \beta)
$$

is a symplectic form on

$$
(0,+\infty) \times L(p, q) .
$$

The condition on $g^{\prime}(t)$ is easily fulfilled as long as $C$ is sufficiently large, so $\Omega$ is the desired symplectic form.

Now consider the diffeomorphism

$$
\begin{aligned}
F: \quad(0,+\infty) \times L(p, q) & \longrightarrow(0,+\infty) \times L(p, q), \\
(t, x) & \longmapsto(t h(x), x) .
\end{aligned}
$$

Then, $F^{*}\left(d\left(t \bar{\alpha}_{0}\right)\right)=d(t \alpha)$. By first gluing via $\psi$ near $\{1\} \times L(p, q)$ and then via $F$ near $\{C+1\} \times L(p, q)$, we obtain $(\widetilde{W}, \widetilde{\omega})$.

Proof of Proposition 3.4. The statement is an immediate consequence of Theorem 3.3, Lemma 3.5 and Lemma 3.6.

Lemma 3.7. Let $p>q \geq 1$ be coprime integers, and suppose that

$$
\frac{p}{p-q}=\left[b_{1}, \ldots, b_{k}\right], \quad b_{1}, \ldots, b_{k} \geq 2 .
$$

Let $N=1+\sum_{i=1}^{k}\left(b_{i}-1\right)$. Then, there exist a symplectic form $\omega$ on

$$
X_{N}:=\mathbb{C P}^{2} \# N \overline{\mathbb{C P}}^{2}
$$

and an embedding

$$
L(p, q) \hookrightarrow X_{N}
$$

which is $\omega$-compatible with respect to the $S^{1}$-action $(3.5)$, such that $X_{N}^{-}$is a compact, regular neighborhood of a symplectic string of type

$$
\left(1,1-b_{1},-b_{2}, \ldots,-b_{k}\right)
$$

in $X_{N}$.

Proof. The proof of the lemma is a simple adaptation of [21, Theorem 2.1 and Corollary 2.1]; therefore we only outline the argument here, and refer to [21] for details. The point is to show that there exist:

(1) an $S^{1}$-Hamiltonian symplectic form $\omega$ on $X_{N}$, and

(2) a Hamiltonian $H: X_{N} \rightarrow \mathbb{R}$ for $\omega$ and a regular value $c \in \mathbb{R}$ of $H$, such that

$$
X^{-}=\{H \geq c\} .
$$

Let $S_{-1}$ be a ruled surface over $\mathbb{C P}^{1}$ with zero section $Z_{0}$ and infinity section $Z_{\infty}$ satisfying $Z_{0} \cdot Z_{0}=-1$ and $Z_{\infty} \cdot Z_{\infty}=1$. There is a standard $S^{1}$-action on $S_{-1}$ which rotates the fibers and fixes $Z_{0}$ and $Z_{\infty}$, and an $S^{1}$-Hamiltonian symplectic structure on $S_{-1}$ with a Hamiltonian function $H: S_{-1} \rightarrow[0,1]$ such that $H\left(Z_{0}\right)=0$ and $H\left(Z_{\infty}\right)=1$. Fix a point $x \in Z_{0}$. The union of $Z_{0}, Z_{1}$ and a fiber of the fibration $S_{-1} \rightarrow \mathbb{C P}^{1}$ through the point $x$ is a symplectic string of type $(-1,0,1)$. Blowing up 
at $x$ and taking proper transforms yields an $S^{1}$-Hamiltonian symplectic structure on $\widehat{S}_{-1}$ together with a symplectic string of type $(-2,-1,-1,1)$. We can keep blowing up at the intersection points of the proper transforms of $Z_{0}$ until the initial part of the string looks like this:

$$
\left(-a_{1},-a_{2}, \ldots,-a_{h},-1, \ldots\right),
$$

where the integers $a_{i} \geq 2$ are chosen so that

$$
\frac{p}{q}=\left[a_{1}, \ldots, a_{h}\right]
$$

Using Riemenschneider's point rule [25] it is easy to check that this can be done so that the whole string is of the following:

$$
\left(-a_{1},-a_{2}, \ldots,-a_{h},-1,-b_{k},-b_{k-1}, \ldots,-b_{2}, 1-b_{1}, 1\right) .
$$

Now the resulting blowup of $S_{-1}$ is $X_{N}$. As explained in the proof of [21, Theorem 2.1] (see also [21, Lemmas 2.3] and [21, Lemmas 2.4]), $X_{N}$ carries an $S^{1}-$ Hamiltonian symplectic form $\omega$ with Hamiltonian

$$
H: X_{N} \rightarrow \mathbb{R},
$$

there is a regular value $c$ of $H$ such that

$$
X^{+}=\{H \leq c\}
$$

is a regular neighborhood of a symplectic string $\Gamma_{1}$ of type

$$
\left(-a_{1}, \ldots,-a_{h}\right)
$$

and

$$
X^{-}=\{H \geq c\}
$$

is a regular neighborhood of a symplectic string $\Gamma_{2}$ of type

$$
\left(1,1-b_{1},-b_{2}, \ldots,-b_{k}\right) .
$$

Moreover, $H^{-1}(c)$ is isomorphic, as an $S^{1}$-manifold, to $L(p, q)$ endowed with the $S^{1}$-action (3.5). This is because the $S^{1}$-action (3.5) is the action induced on $L(p, q)$ when the lens space is viewed as the boundary of the $S^{1}$-equivariant plumbing determined by the weighted graph dual to $\Gamma_{1}$, which coincides, by [21, Corollary 2.1], with the $S^{1}$-action induced on $H^{-1}(c)$.

Proof of Theorem 3.2. By Lemma 3.7 we can find a symplectic structure $\omega$ on a rational symplectic four-manifold $X$ and an embedding $L(p, q) \hookrightarrow X$ which is $\omega$-compatible with respect to the $S^{1}$-action (3.5) and such that $X^{-}$is a regular neighborhood of a symplectic string

$$
\widetilde{\Gamma}=\widetilde{C}_{0} \cup \widetilde{C}_{1} \cup \cdots \widetilde{C}_{k} \subset X
$$

of type $\left(1,1-b_{1},-b_{2}, \ldots,-b_{k}\right)$. Applying Proposition 3.4 we can construct a symplectic manifold of the form

$$
\left(X_{W}=W \cup_{\partial W=\partial X^{-}} X^{-}, \bar{\omega}\right) .
$$

By [19], if $(M, \omega)$ is a closed symplectic four-manifold containing an embedded symplectic two-sphere $C$ of self-intersection +1 such that $M \backslash C$ is minimal, then $(M, \omega)$ is symplectomorphic to $\mathbb{C P}^{2}$ with the standard Kähler form. Moreover, the symplectomorphism can be chosen such that it sends $C$ to a complex line. 
Since $\widetilde{C}_{0} \subset X_{W}$ has self-intersection +1 , and since non-minimal symplectic fourmanifolds can be reduced to minimal ones by blowing down exceptional symplectic spheres, we conclude that, for some $M \geq 0$, there is a symplectomorphism

$$
\psi:\left(X_{W}, \bar{\omega}\right) \rightarrow\left(\mathbb{C P}^{2} \# M \overline{\mathbb{C P}}^{2}, \omega_{M}\right)
$$

sending $\widetilde{C}_{0}$ to a complex line $C_{0} \subset \mathbb{C P}^{2}$. Clearly,

$$
\Gamma=C_{0} \cup \cdots \cup C_{k}=\psi(\widetilde{\Gamma}) \subset \mathbb{C P}^{2} \# M \overline{\mathbb{C P}}^{2}
$$

is a symplectic string of type $\left(1,1-b_{1},-b_{2}, \ldots,-b_{k}\right)$.

\section{Complements of Symplectic Strings}

The purpose of this section is to prove Theorem 1.1(1), which follows immediately from Theorem 3.2 combined with the following result:

Theorem 4.1. Let $\omega_{M}$ be a symplectic form on $X_{M}=\mathbb{C P}^{2} \# M \overline{\mathbb{C P}}^{2}$ obtained from the standard Kähler form on $\mathbb{C P}^{2}$ by symplectic blowups. Let

$$
\Gamma=C_{0} \cup C_{1} \cup \cdots \cup C_{k} \subset X_{M}
$$

be a symplectic string of type

$$
\left(1,1-b_{1},-b_{2}, \ldots,-b_{k}\right), \quad k \geq 2, \quad b_{1}, \ldots, b_{k} \geq 1,
$$

and such that $C_{0} \subset \mathbb{C P}^{2}$ is a complex line. Let $s_{i}$, for $i=1, \ldots, k$, denote one half of the cardinality of the set

$S_{i}:=\left\{e \in H_{2}\left(X_{M} ; \mathbb{Z}\right) \mid e \cdot e=-1, e \cdot\left[C_{i}\right] \neq 0, e \cdot\left[C_{j}\right]=0\right.$ for $\left.j \in\{0, \ldots, k\} \backslash\{i\}\right\}$ and let $n_{i}:=b_{i}-s_{i}$. Then, $\mathbf{n}:=\left(n_{1}, \ldots, n_{k}\right) \in \mathbf{Z}_{p, q}$, and the complement of a regular neighborhood of $\Gamma$ is orientation preserving diffeomorphic to a smooth blowup of $W_{p, q}(\mathbf{n})$.

Theorem 4.1 will follow from Theorem 4.2 below.

Theorem 4.2. Let $\omega_{M}$ be a symplectic form on $X_{M}=\mathbb{C P}^{2} \# M \overline{\mathbb{C P}}^{2}$ obtained from the standard Kähler form on $\mathbb{C P}^{2}$ by symplectic blowups. Let

$$
\Gamma=C_{0} \cup C_{1} \cup \cdots \cup C_{k} \subset X_{M}
$$

be a symplectic string of type

$$
\left(1,1-b_{1},-b_{2}, \ldots,-b_{k}\right), \quad k \geq 2, \quad b_{1}, \ldots, b_{k} \geq 1,
$$

and such that $C_{0} \subset \mathbb{C P}^{2}$ is a complex line. Then, there is a sequence of symplectic blowdowns

$$
\left(\mathbb{C P}^{2} \# M \overline{\mathbb{C P}}^{2}, \omega_{M}\right) \rightarrow\left(\mathbb{C P}^{2} \#(M-1) \overline{\mathbb{C P}}^{2}, \omega_{M-1}\right) \rightarrow \cdots \rightarrow\left(\mathbb{C P}^{2}, \omega_{0}\right)
$$

with $\omega_{0}$ diffeomorphic to the standard Kähler form and such that $\Gamma$ descends to a symplectic string of type $(1,1)$ in $\left(\mathbb{C P}^{2}, \omega_{0}\right)$.

Before proving Theorems 4.1 and 4.2 we need to establish some auxiliary results.

Lemma 4.3. Let $\left\{l, f_{1}, \ldots, f_{M}\right\} \subseteq H_{2}\left(\mathbb{C P}^{2} \# M \overline{\mathbb{C P}}^{2} ; \mathbb{Z}\right)$ be a set of generators which are orthogonal with respect to the intersection form, with $l$ being the homology class of a complex line in $\mathbb{C P}^{2}$ and with each $f_{i}$ having square -1 . Let $\alpha_{1}, \ldots, \alpha_{k} \in$ $H_{2}\left(\mathbb{C P}^{2} \# M \overline{\mathbb{C P}}^{2} ; \mathbb{Z}\right), k \geq 2$, be homology classes of the form

$$
\alpha_{1}=l-e_{1}^{1}-e_{2}^{1}-\cdots-e_{b_{1}}^{1}, \quad \alpha_{i}=e_{1}^{i}-e_{2}^{i}-\cdots-e_{b_{i}}^{i}, \quad i=2, \ldots, k,
$$


where $b_{i} \geq 1$ for $i=1, \ldots, k, e_{j}^{i} \in\left\{f_{1}, \ldots, f_{M}\right\}$ for every $i, j$ and $e_{j}^{i} \neq e_{j^{\prime}}^{i}$ if $j \neq j^{\prime}$. Suppose also that

$$
\alpha_{i} \cdot \alpha_{j}= \begin{cases}1 & \text { if }|i-j|=1 \\ 0 & \text { if }|i-j|>1\end{cases}
$$

and let $A^{1}=\left\{e_{1}^{1}, \ldots, e_{b_{1}}^{1}\right\}$ and $A^{i}=\left\{e_{2}^{i}, \ldots, e_{b_{i}}^{i}\right\}$ for $i=2, \ldots, k$. Then,

(1) for every $j=2, \ldots, k$, there is an index $i$ with $1 \leq i<j$ such that

$$
e_{1}^{j} \in A^{i}
$$

Moreover, if $e_{1}^{j} \in A^{i}$ with $i<j-1$,

$$
e_{1}^{h} \in A^{i} \cap A^{j}
$$

for some index $h$ with $i<h<j$;

(2) for every $1 \leq i<j \leq k$ we have

$$
A^{i} \cap A^{j} \subseteq\left\{e_{1}^{2}, \ldots, e_{1}^{k}\right\} .
$$

Proof. (1) We argue by induction on $k \geq 2$. For $k=2$, (1) applies only to $j=2$, in which case the statement clearly holds because $\alpha_{1} \cdot \alpha_{2}=1$.

Now suppose that $k>2$ and the statement to be true for $\alpha_{1}, \ldots, \alpha_{k-1}$. Clearly, it suffices to prove the statement for $j=k$. Since $\alpha_{k-1} \cdot \alpha_{k}=1$, either $e_{1}^{k} \in A^{k-1}$, in which case we are done, or $e_{1}^{k-1} \in A^{k}$. In the latter case, we set $j_{1}=k-1$, and the induction hypothesis implies $e_{1}^{k-1} \in A^{j_{2}}$ for some $j_{2}<j_{1}$. Thus, $A^{j_{2}} \cap A^{k} \neq \emptyset$ and, since $\alpha_{j_{2}} \cdot \alpha_{k}=0$, we either have $e_{1}^{k} \in A^{j_{2}}$, in which case, setting $i=j_{2}$ and $h=k-1$, we are done because $e_{1}^{k-1} \in A^{j_{2}} \cap A^{k}$ or $e_{1}^{j_{2}} \in A^{k}$. Continuing in this fashion we obtain a maximal, strictly decreasing sequence of indexes

$$
k=j_{0}>k-1=j_{1}>j_{2}>\cdots>j_{r} \geq 1
$$

such that $e_{1}^{j_{s}} \in A^{j_{s+1}} \cap A^{k}$ for $s=1, \ldots, r-1$. Then, $e_{1}^{k} \in A^{j_{r}}$. In fact, if $e_{1}^{k} \notin A^{j_{r}}$, then, since $\alpha_{1} \cdot \alpha_{k}=0$, we would have $j_{r}>1$; therefore the sequence could be extended, contradicting its maximality. This proves (1) setting $i=j_{r}$ and, when $j_{r}<k-1, h=j_{r-1}$.

(2) The proof is again an induction on $k \geq 2$. For $k=2, \alpha_{1} \cdot \alpha_{2}$ implies $A^{1} \cap A^{2}=\emptyset$, so suppose $k>2$ and the statement to be true for $\alpha_{1}, \ldots, \alpha_{k-1}$.

Observe that, since $\alpha_{i} \cdot \alpha_{k} \geq 0$ for $i<k$,

$$
e_{1}^{k} \notin\left\{e_{1}^{1}, \ldots, e_{1}^{k-1}\right\} .
$$

This implies that $e_{1}^{k}$ belongs to at most one of the sets $A^{1}, A^{2}, \ldots, A^{k-1}$, because $e_{1}^{k} \in A^{i} \cap A^{j}$ for $1 \leq i<j \leq k-1$ implies

$$
A^{i} \cap A^{j} \not\left\{\left\{e_{1}^{2}, \ldots, e_{1}^{k-1}\right\},\right.
$$

contrary to the induction hypotheses.

Arguing by contradiction, suppose the statement to be false for $\alpha_{1}, \ldots, \alpha_{k}$. By the induction hypothesis, for some $1 \leq i \leq k-1$ we have

$$
A^{i} \cap A^{k} \nsubseteq\left\{e_{1}^{2}, \ldots, e_{1}^{k}\right\} .
$$

We claim that $i \neq k-1$. In fact, if $i=k-1$, then $\alpha_{k-1} \cdot \alpha_{k}=1$ and $A^{k-1} \cap A^{k} \neq \emptyset$ imply $e_{1}^{k} \in A^{k-1}$ and $e_{1}^{k-1} \in A^{k}$. But by (1) there is an index $i^{\prime}<k-1$ such that $e_{1}^{k-1} \in A^{i^{\prime}}$. Thus, since $\alpha_{i^{\prime}} \cdot \alpha_{k}=0, e_{1}^{k} \in A^{k-1}$, and by the induction hypothesis

$$
A^{i^{\prime}} \cap A^{k-1} \subseteq\left\{e_{1}^{2}, \ldots, e_{1}^{k-1}\right\}
$$


we have $e_{1}^{h} \in A^{k}$. Now we can apply (1) again with $j=i^{\prime}$ and argue in the same way. After a finite number of similar steps we are forced to conclude $e_{1}^{k} \in A^{1}$, which is incompatible with $e_{1}^{k} \in A^{k-1}$.

We can now finish the proof assuming $i \neq k-1$. Since $\alpha_{i} \cdot \alpha_{k}=0$, either $e_{1}^{i} \in A^{k}$ or $e_{1}^{k} \in A^{i}$. In the latter case, by (1) we have $e_{1}^{h} \in A^{i} \cap A^{k}$ for some index $h$ with $i<h<k$. But $A^{i} \cap A^{k} \nsubseteq\left\{e_{1}^{2}, \ldots, e_{1}^{k}\right\}$ implies that $A^{i} \cap A^{k}$ has at least two elements. Therefore, in any case we have $e_{1}^{i} \in A^{k}$. Repeated applications of (1) starting with $j=i$ and $j=k-1$ respectively, yield two maximal strictly decreasing sequences

$$
i=h_{0}>h_{1}>\cdots>h_{r} \geq 1, \quad k-1=l_{0}>l_{1}>\cdots>l_{s} \geq 1,
$$

such that

$$
e_{1}^{h_{n}} \in A^{h_{n+1}} \cap A^{k}, \quad n=0, \ldots, r-1,
$$

and

$$
e_{1}^{l_{m}} \in A^{l_{m+1}} \cap A^{k}, \quad m=0, \ldots, s-1 .
$$

Maximality implies $e_{1}^{h_{r}} \notin A^{k}$ if $h_{r}>1, e_{1}^{l_{s}} \notin A^{k}$ if $l_{s}>1$, and $e_{1}^{k} \in A^{h_{r}} \cap A^{l_{s}}$. Since $e_{1}^{k}$ belongs to at most one of the sets $A^{1}, \ldots, A^{k-1}$, we must have $h_{r}=l_{s}$. Therefore there exist $\bar{n}$ with $0 \leq \bar{n}<r$ and $\bar{m}$ with $0 \leq \bar{m}<s$ such that $h_{\bar{n}} \neq l_{\bar{m}}$ but $h_{\bar{n}+1}=l_{\bar{m}+1}$. This implies that each of the distinct elements $e_{1}^{h_{\bar{n}}}$ and $e_{1}^{l_{\bar{m}}}$ belong to $A^{h_{\pi+1}} \cap A^{k}$. Since $\alpha_{h_{\pi+1}} \cdot \alpha_{k}=0$, we must have $e_{1}^{h_{\bar{n}+1}} \in A^{k}, e_{1}^{k} \in A^{h_{\pi+1}}$, and therefore $r=\bar{n}+1=\bar{m}+1=s$, which is incompatible with $e_{1}^{h_{r}} \notin A^{k}$.

Proposition 4.4. Let $X_{M}=\mathbb{C P}^{2} \# M \overline{\mathbb{C P}}^{2}$ be endowed with a blowup $\omega_{M}$ of the standard Kähler form on $\mathbb{C P}^{2}$, and let

$$
\Gamma=C_{0} \cup C_{1} \cup \cdots \cup C_{k} \subset X_{M}
$$

be a symplectic string of type

$$
\left(1,1-b_{1},-b_{2}, \ldots,-b_{k}\right), \quad b_{1}, \ldots, b_{k} \geq 1 .
$$

Let $\left\{l, f_{1}, \ldots, f_{M}\right\} \subseteq H_{2}\left(\mathbb{C P}^{2} \# M \overline{\mathbb{C P}}^{2} ; \mathbb{Z}\right)$ be a set of generators which are orthogonal with respect to the intersection form, with l being the homology class of a complex line in $\mathbb{C P}^{2}$ and with each $f_{i}$ having square -1 . Suppose that $\left[C_{0}\right] \in H_{2}\left(X_{M} ; \mathbb{Z}\right)$ is equal to the homology class $l$. Then,

$$
\left[C_{1}\right]=l-e_{1}^{1}-e_{2}^{1}-\cdots-e_{b_{1}}^{1}, \quad\left[C_{i}\right]=e_{1}^{i}-e_{2}^{i}-\cdots-e_{b_{i}}^{i}, \quad i=2, \ldots, k,
$$

where $e_{j}^{i} \in\left\{f_{1}, \ldots, f_{M}\right\}$ for every $i, j$ and $e_{j}^{i} \neq e_{j^{\prime}}^{i}$ if $j \neq j^{\prime}$.

Proof. We can write

$$
\left[C_{i}\right]=\delta_{1 i} l+\sum_{j=1}^{M} a_{j}^{i} f_{j}
$$

for some coefficients $a_{j}^{i} \in \mathbb{Z}, i=1, \ldots, k$, where $\delta_{1 i}$ is Krönecker's delta. Since each $C_{i}$ is symplectic,

$$
\left\langle c_{1}\left(X_{M}\right),\left[C_{i}\right]\right\rangle=2+C_{i} \cdot C_{i} \quad \text { for } \quad i=1, \ldots, k,
$$

which is equivalent to:

$$
\sum_{j=1}^{M}\left(a_{j}^{i}+\left(a_{j}^{i}\right)^{2}\right)=2\left(1-\delta_{1 i}\right) .
$$


We assume $k \geq 1$ and prove the statement by induction on $k$. Equation (4.2) implies immediately that $a_{j}^{1} \in\{0,-1\}$ for $j=1, \ldots, M$. Therefore, the statement holds for $k=1$.

Now suppose that $k \geq 2$ and that the classes $\left[C_{i}\right]$ have the form given in the statement for $i=1, \ldots, k-1$. By Equation (4.2) for $i=k$, there is exactly one index $j_{0} \in\{1, \ldots, M\}$ such that $a_{j_{0}}^{k} \in\{1,-2\}$, while $a_{j}^{k} \in\{0,-1\}$ for $j \neq j_{0}$.

We claim that the equality $a_{j_{0}}^{k}=-2$ leads to a contradiction. In fact, since $C_{k} \cdot C_{k-1}=1$, if all the coefficients $a_{j}^{k}$ are non-positive, then we must have $k>2$ and $a_{1}^{k-1} \in A^{k}$. By Lemma 4.3(1) applied for $j=k-1, e_{1}^{k-1} \in A^{i}$ for some $i<k-1$. Since $C_{i} \cdot C_{k}=0$ and all the coefficients $a_{j}^{k}$ are non-positive, we must have $i>1$ and $a_{1}^{i} \in A^{k}$. Now we can apply Lemma 4.3(1) again for $j=i$, and argue in the same way. Clearly, after a finite number of similar steps we reach a contradiction.

Lemma 4.5. Under the assumptions of Theorem 4.2, let $J$ be an almost complex structure tamed by $\omega_{M}$ and such that $\Gamma$ is J-holomorphic. Then, there exists an embedded $J$-holomorphic sphere $\Sigma \subset X_{M}$ such that $[\Sigma] \cdot\left[C_{0}\right]=0$ and $[\Sigma] \cdot[\Sigma]=$ -1 . Moreover, there exists such a $\Sigma$ disjoint from $\Gamma$ if and only if there exists a symplectic sphere $S \subset X_{M}$ of square -1 such that $[S] \cdot\left[C_{i}\right]=0$ for $i=0, \ldots, k$.

Proof. Since the symplectic $4-$ manifold $X_{M}$ is obtained by blowing up $\mathbb{C P}^{2}$, there exists a symplectic sphere $S \subset X_{M}$ of square -1 orthogonal to $l=\left[C_{0}\right]$. By [19, Lemma 3.1], the homology class $[S]$ is either represented by an embedded $J-$ holomorphic sphere $\Sigma$ or by a $J$-holomorphic cusp-curve

$$
S_{1} \cup \cdots \cup S_{n},
$$

i.e. a union of (not necessarily embedded) $J$-holomorphic spheres. In the first case, the first part of the lemma is proved. In the second case notice that, since $[S] \cdot\left[C_{0}\right]=0$, by positivity of intersections $[20]$ we have

$$
\left[S_{i}\right] \cdot\left[C_{0}\right]=0 \text { for } i=1, \ldots, n .
$$

Therefore

$$
\left[S_{i}\right] \cdot\left[S_{i}\right] \leq-1 \text { for } i=1, \ldots, n
$$

Moreover,

$$
1=\chi(S)+S \cdot S=\left\langle c_{1}\left(X_{M}\right),[S]\right\rangle=\sum_{i=1}^{n}\left\langle c_{1}\left(X_{M}\right),\left[S_{i}\right]\right\rangle=\sum_{i=1}^{n}\left(\chi\left(S_{i}\right)+\left[S_{i}\right] \cdot\left[S_{i}\right]\right),
$$

which implies $\left[S_{j}\right] \cdot\left[S_{j}\right]=-1$ for at least one index $j \in\{1, \ldots, n\}$. By the adjunction formula [19], $\Sigma:=S_{j}$ is embedded. Hence, the first part of the lemma is proved. If $[S]$ is orthogonal to all the classes $\left[C_{i}\right]$, then by positivity of intersections so is $[\Sigma]$, and therefore $\Sigma$ must be disjoint from $\Gamma$. This proves the second part of the lemma.

Proof of Theorem 4.2. Let $J$ be an almost complex structure tamed by $\omega_{M}$ and such that $\Gamma$ is $J$-holomorphic. If there exists an embedded symplectic sphere $S \subset$ $X_{M}$ such that $[S] \cdot\left[C_{i}\right]=0, i=0, \ldots, k$, then by Lemma 4.5 there is an embedded $J$-holomorphic sphere $\Sigma \subset X_{M} \backslash \Gamma$ with self-intersection $\Sigma \cdot \Sigma=-1$. Therefore, we may blow down $\Sigma$ and reapply the same argument to $X_{M-1}$. After a finite number of similar steps we get $\Gamma \subset X_{M^{\prime}}$, with the property that for every symplectic sphere $S \subset X_{M}$ the class $[S]$ intersects non-trivially at least one of the classes $\left[C_{i}\right]$. 
Applying Lemma 4.5 again, we know that there exists an embedded $J$-holomorphic sphere $\Sigma \subset X_{M^{\prime}}$ such that $[\Sigma] \cdot\left[C_{0}\right]=0$ and $\Sigma \cap \Gamma \neq \emptyset$. By Proposition 4.4, the homology classes $\left[C_{i}\right]$ have the form $(4.1)$. Since $[\Sigma] \cdot\left[C_{0}\right]=0,[\Sigma]$ must coincide with one of the classes $e_{j}^{i}$.

Now we argue by induction on $k \geq 2$. Clearly, either for some $s \geq 2$ we have $b_{s}=1$ and $[\Sigma]=\left[C_{s}\right]$, or $[\Sigma] \cdot C_{i} \in\{0,1\}$ for every $i=1, \ldots, k$.

If $b_{s}=1$ and $[\Sigma]=\left[C_{s}\right]$, by positivity of intersections we must have $\Sigma=C_{s}$. In this case we can blow down $\Sigma$, and $\Gamma$ descends to a symplectic string $\Gamma_{1} \subset X_{M^{\prime}-1}$ of length strictly less than the length of $\Gamma$. If $k=2$, then $s=2, b_{1}=b_{2}=1$ and $\Gamma_{1}=C_{0} \cup C_{1}^{\prime}$ is a symplectic string of type $(1,1)$ with $\left[C_{1}^{\prime}\right]=l$. Since the complement of $C_{0}$ is minimal, the conclusion follows immediately from the results of [19] as in the proof of Theorem 3.2. If $k>2$, since the intersection form of $X_{M^{\prime}-1}$ restricted to the orthogonal complement of the class $l$ is negative definite, $\Gamma_{1}$ satisfies the hypothesis of the theorem. By induction, the statement holds for $\Gamma_{1}$ and therefore for $\Gamma$.

If $[\Sigma] \cdot C_{i} \in\{0,1\}$ for every $i=1, \ldots, k$, we must have $\Sigma \cdot C_{i}=1$ for at least one index $i>0$. By Lemma 4.3(2), in this case there is exactly one such index, so $\Sigma$ must intersect $\Gamma \backslash C_{0}$ transversely at one smooth point. As before, if we blow down $\Sigma$, then $\Gamma$ descends to a symplectic string $\Gamma_{1} \subset X_{M^{\prime}-1}$ satisfying the hypothesis of the theorem. Now we can go through the same process starting from the beginning, thus showing that $\Gamma_{1}$ descends to a symplectic string $\Gamma_{2} \subset X_{M^{\prime}-2}$. After a finite number of similar steps we arrive at a symplectic string of length strictly less than the length of $\Gamma$, and the induction argument works as before.

Proof of Theorem 4.1. By Theorem 4.2, there is a sequence of symplectic blowdowns

$$
\left(\mathbb{C P}^{2} \# M \overline{\mathbb{C P}}^{2}, \omega_{M}\right) \rightarrow\left(\mathbb{C P}^{2} \#(M-1) \overline{\mathbb{C P}}^{2}, \omega_{M-1}\right) \rightarrow \cdots \rightarrow\left(\mathbb{C P}^{2}, \omega_{0}\right)
$$

with $\omega_{0}$ diffeomorphic to the standard Kähler form, and such that $\Gamma$ descends to a symplectic string of type $(1,1)$ in $\left(\mathbb{C P}^{2}, \omega_{0}\right)$. Since we want to determine the complement of a regular neighborhood of $\Gamma$ up to diffeomorphisms, we may assume that $\omega_{0}$ is the standard Kähler form on $\mathbb{C P}^{2}$.

Let $J_{0}$ be an almost complex structure in $\mathbb{C P}^{2}$ tamed by $\omega_{0}$ and such that $l_{0}$ and $l_{0}^{\prime}$ are $J_{0}$-holomorphic. The results of [12] show that if $\left\{J_{t}\right\}_{t \in[0,1]}$ is a generic path of tamed almost complex structures connecting $J_{0}$ to the standard complex structure $J_{1}$, then there are families $\left\{l_{t}\right\}_{t \in[0,1]}$ and $\left\{l_{t}^{\prime}\right\}_{t \in[0,1]}$ of smooth, embedded and distinct $J_{t}$-holomorphic spheres connecting $l_{0}$, respectively $l_{0}^{\prime}$, to standard complex lines $l_{1}$ and $l_{1}^{\prime}$. This shows that the string $l_{0} \cup l_{0}^{\prime}$ is symplectically isotopic to a pair $l_{1} \cup l_{1}^{\prime}$ of distinct standard complex lines.

Thus, in order to determine the diffeomorphism type of the complement of a regular neighborhood of $\Gamma$, one may replace $\Gamma$ with the proper transform of a pair of distinct complex lines in $\mathbb{C P}^{2}$ under the sequence of complex blowups corresponding to (4.3). In fact, by analyzing the construction of Sequence (4.3) in the proof of Theorem 4.2 and using Kirby calculus and Lemma 2.2, it is easy to see that there is an orientation preserving diffeomorphism

$$
W \cong W_{p, q}(\mathbf{n}) \# r \overline{\mathbb{C P}}^{2},
$$

with $\mathbf{n} \in \mathbf{Z}_{p, q}$ as in the statement and $r=M-\sum_{i=1}^{k}\left(b_{i}-n_{i}\right)$. 


\section{Stein structures on $W_{p, q}(\mathbf{n})$}

In this section we construct Stein structures on the smooth four-manifolds with boundary $W_{p, q}(\mathbf{n})$ defined in Section 1 . The proof is based on Legendrian surgery [4, $11]$.

A knot $K$ in a contact three-manifold $(Y, \xi)$ is called Legendrian if $K$ is everywhere tangent to the distribution $\xi$. The contact structure induces a framing of $K$, usually called the contact framing and denoted by $\operatorname{tb}(K)$. Assume that $\xi$ is oriented. Given an oriented Legendrian knot $K$ in $(Y, \xi)$ and a non-zero section $v$ of $\xi$ along $K$, the rotation number $\operatorname{rot}_{v}(K)$ is the winding number of the oriented tangent vector to $K$ with respect to $v$ in the oriented plane $\xi$.

Let $\left(n_{1}, \ldots, n_{k}\right)$ be an admissible sequence of positive integers such that

$$
\left[n_{1}, \ldots, n_{k}\right]=0 .
$$

We fix once and for all a sequence of strict blowdowns

$$
\left(n_{1}, \ldots, n_{k}\right) \rightarrow \cdots \rightarrow(0)
$$

as in Lemma 2.2. We can realize this sequence of operations geometrically, by viewing each step as a blowdown in the sense of the Kirby calculus on framed links, starting from the framed link $L=\bigcup_{i=1}^{k} L_{i}$ of Figure 1 and ending with the zero-framed unknot. Such a sequence corresponds to an orientation preserving diffeomorphism

$$
\varphi: N(\mathbf{n}) \rightarrow S^{1} \times S^{2},
$$

where $N(\mathbf{n})=N\left(n_{1}, \ldots, n_{k}\right)$ is the closed oriented three-manifold obtained by surgery along the framed link $L \subset S^{3}$. Let $\nu(L) \subset S^{3}$ be a small tubular neighborhood of $L$. Then, the complement $S^{3} \backslash \nu(L)$ can be identified with a subset $\mathcal{C}$ of $N(\mathbf{n})$, i.e. the complement of the surgered solid tori. Every link in $\mathcal{C} \subset S^{3}$ is therefore endowed with a canonical framing. We shall use this canonical framing to identify any framing with a $k$-uple of integers.

The smooth manifolds $W_{p, q}(\mathbf{n})$ are obtained by first attaching a one-handle to the four-ball, and then attaching two-handles to the boundary of the resulting $S^{1} \times D^{3}$. The standard tight contact structure $\zeta_{0}$ on $S^{1} \times S^{2}=\partial\left(S^{1} \times D^{3}\right)$ is obtained from the standard contact structure $\xi_{\text {st }}$ on $S^{3}$ by removing two smooth balls and gluing the resulting boundaries in a suitable way. Moreover, each Legendrian link in $\left(S^{1} \times S^{2}, \zeta_{0}\right)$ is contact isotopic to a Legendrian link in standard form in the sense of [11]. In particular, there is a nowhere vanishing section of $\zeta_{0}$, denoted by $\frac{\partial}{\partial x}$ in [11], along any Legendrian link in standard form. The section $\frac{\partial}{\partial x}$ extends as a nowhere vanishing section $v$ of $\zeta_{0}$ to all of $S^{1} \times S^{2}$. The contact structure $\zeta_{0}$ has a natural orientation coming from the natural complex orientation on $\xi_{\text {st }}$. Therefore, the rotation numbers with respect to the section $v$ are well-defined.

Using Eliashberg's Legendrian surgery construction $[4,11]$, we will now prove that there are Stein structures on each $W_{p, q}(\mathbf{n})$ by showing that the attaching circles of the two-handles are isotopic to Legendrian knots

$$
K_{i} \subset\left(S^{1} \times S^{2}, \zeta_{0}\right)
$$

and the two-handles are attached with framings $\operatorname{tb}\left(K_{i}\right)-1$.

Let $\widetilde{\zeta}_{0}=\varphi^{*}\left(\zeta_{0}\right)$ be the tight contact structure on $N(\mathbf{n})$ obtained by pulling back $\zeta_{0}$ via the diffeomorphism $(5.2)$. Observe that $\widetilde{\zeta}_{0}$ does not depend on the choice of 
$\varphi$ because $S^{1} \times S^{2}$ carries only one tight contact structure up to isotopy. Let $\tau$ be the pull-back of the nowhere zero section $v$ by the diffeomorphism (5.2).

Recall from Section 1 that, given two coprime integers

$$
p>q \geq 1, \quad \text { with } \quad \frac{p}{p-q}=\left[b_{1}, \ldots, b_{k}\right],
$$

we defined the set

$$
\mathbf{Z}_{p, q}=\left\{\left(n_{1}, \ldots, n_{k}\right) \in \mathbb{Z}^{k} \mid\left[n_{1}, \ldots, n_{k}\right]=0,0 \leq n_{i} \leq b_{i}, i=1, \ldots, k\right\} .
$$

Theorem 5.1. Let $p>q \geq 1$ be coprime integers with $\frac{p}{p-q}=\left[b_{1}, \ldots, b_{k}\right]$, and let $\mathbf{n}=\left(n_{1}, \ldots, n_{k}\right) \in \mathbf{Z}_{p, q}$. Let

$$
\mathbf{L}=\bigcup_{i=1}^{k} \mathbf{L}_{i} \subset \mathcal{C}=S^{3} \backslash \nu(L)
$$

be the "thick" link drawn in Figure 3. Then, there exists a Legendrian link

$$
\mathbf{L}=\bigcup_{i=1}^{k} \mathcal{L}_{i} \subset\left(\mathcal{C},\left.\widetilde{\zeta}_{0}\right|_{\mathcal{C}}\right)
$$

with the following properties:

(a) $\mathcal{L}$ is smoothly isotopic to $\mathbf{L}$ inside $\mathcal{C}$.

(b) $\mathcal{L}$ has contact framing equal to the canonical framing induced by the inclusion $\mathcal{C} \subset S^{3}$.

(c) Define $\operatorname{rot}_{\tau}\left(\mathcal{L}_{i}\right)$ to be 0 for $i<1$ and $i>k$. Then, $\mathcal{L}$ admits an orientation such that $\operatorname{rot}_{\tau}\left(\mathcal{L}_{1}\right)=0$, and

$$
\operatorname{rot}_{\tau}\left(\mathcal{L}_{i-1}\right)+\operatorname{rot}_{\tau}\left(\mathcal{L}_{i+1}\right)-n_{i} \operatorname{rot}_{\tau}\left(\mathcal{L}_{i}\right)=\left\{\begin{aligned}
1 & \text { if } i=1, \\
0 & \text { if } 1<i<k, \\
-1 & \text { if } i=k .
\end{aligned}\right.
$$
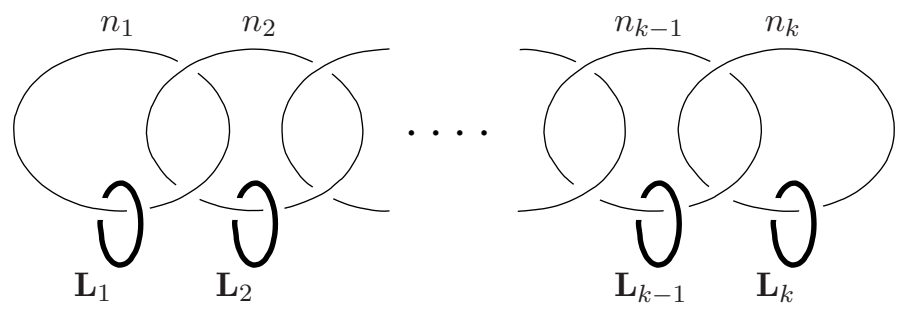

FiguRE 3. The link $\mathbf{L}$

Proof. We argue by induction on $k \geq 1$. When $k=1$ we have $\frac{p}{p-q}=\left[b_{1}\right]$ and $\left[n_{1}\right]=0$, therefore $b_{1}=p, q=p-1, n_{1}=0$ and $\varphi$ is the identity. The formulas for the Thurston-Bennequin and the rotation number of a Legendrian link in standard form [11] show that the Legendrian link $\mathcal{L}$ of Figure 4 satisfies (a), (b) and (c). Now assume $k>1$, and suppose that the statement holds for every four-manifold of the form $W_{p^{\prime}, q^{\prime}}(\mathbf{m})$, where $\mathbf{m}=\left(m_{1}, \ldots, m_{k-1}\right)$. Let

$$
\mathbf{n}=\left(n_{1}, \ldots, n_{s-1}, 1, n_{s+1}, \ldots, n_{k}\right) \rightarrow \mathbf{n}^{\prime}=\left(n_{1}, \ldots, n_{s-1}-1, n_{s+1}-1, \ldots, n_{k}\right)
$$




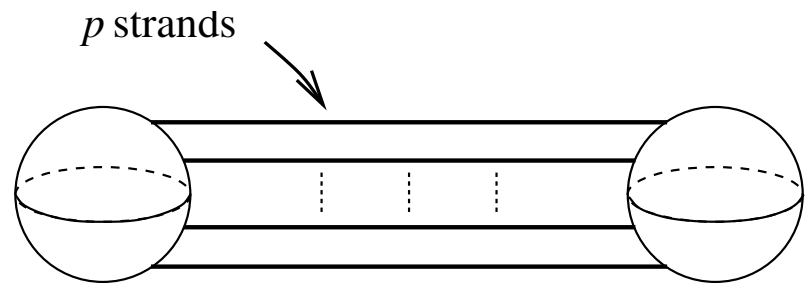

Figure 4. The link $\mathcal{L}$ when $k=1$

be the first element of the sequence of strict blowdowns (5.1). By definition of a strict blowdown, $s>1$. Redraw Figure 3 as Figure 5 , where the thin horizontal arcs in the picture are the strands of the braid $\beta=\sigma_{1}^{2} \sigma_{2}^{2} \cdots \sigma_{k-1}^{2} \in B_{k}$. Observe that the closure of $\beta$ is isotopic to the thin link $L$ of Figure 3. We orient $\mathbf{L}$ as shown in Figure 5.

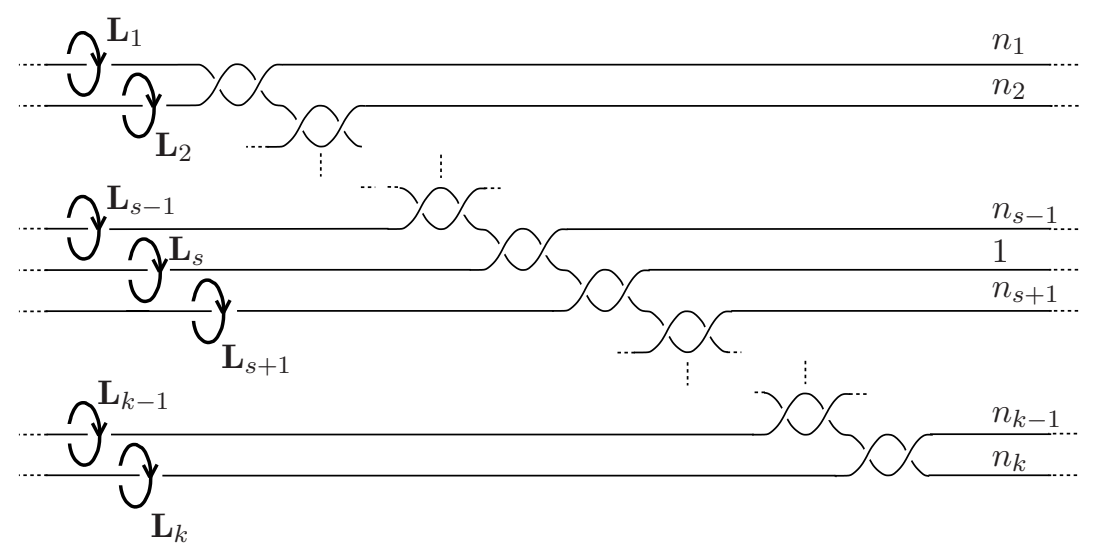

FiguRE 5. The link $\mathbf{L}$ redrawn and oriented

We now prove the statement assuming $s \neq k$, which is the harder case. At the end we will briefly say how to deal with the easier case $s=k$, omitting some obvious details.

Blowing down the $s$-th component of the thin link $L$ yields Figure 6 . The resulting thick link $\widetilde{\mathbf{L}}$ is the disjoint union of $\mathbf{L}^{\prime}$ and $\mathbf{L}_{s}^{\prime \prime}$, where $\mathbf{L}^{\prime}=\bigcup_{i \neq s} \mathbf{L}_{i}^{\prime}$ is the collection of thick unknots, each of which is linked to a single strand of the thin braid, on the left-hand side of the picture, and $\mathbf{L}_{s}^{\prime \prime}$ is the only thick unknot which links two strands. We can view the link $\widetilde{\mathbf{L}}$ as sitting inside the oriented three-manifold $N\left(\mathbf{n}^{\prime}\right)$ obtained by surgery in $S^{3}$ along the thin framed link of Figure 6 , which we call $L^{\prime}$. The diffeomorphism $\varphi$ of $(5.2)$ is the composition of two diffeomorphisms:

$$
N(\mathbf{n}) \stackrel{\psi}{\longrightarrow} N\left(\mathbf{n}^{\prime}\right) \stackrel{\varphi^{\prime}}{\longrightarrow} S^{1} \times S^{2} .
$$

Here $\psi$ is determined by the first element in the sequence (5.1), and $\varphi^{\prime}$ by the remaning elements. Let $\widetilde{\zeta}_{0}^{\prime}$ be the pull-back to $N\left(\mathbf{n}^{\prime}\right)$ of $\zeta_{0}$ via $\varphi^{\prime}$. Observe that the oriented knot $\mathbf{L}_{s}^{\prime \prime}$ is isotopic to an oriented band connected sum of the oriented knots 


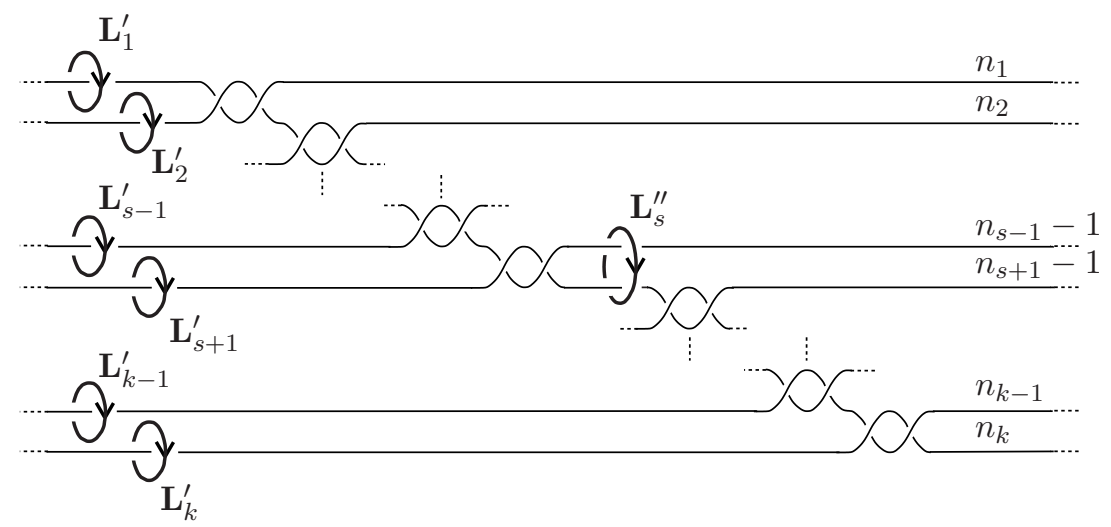

FiguRE 6. The link L blown down

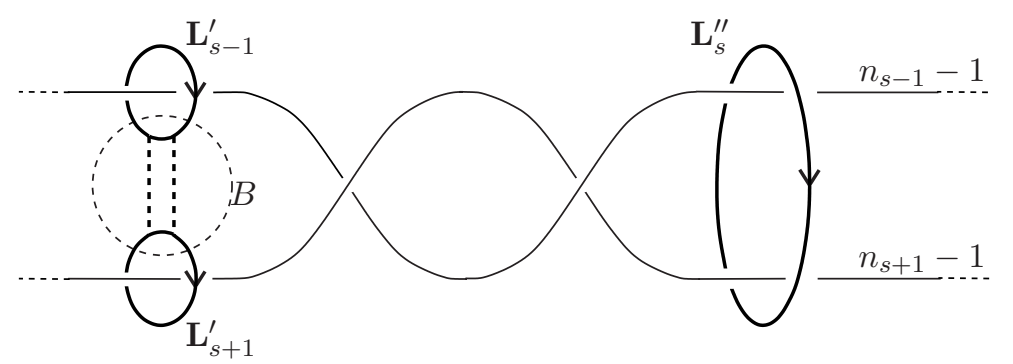

Figure 7 . The band connected sum

$\mathbf{L}_{s-1}^{\prime}$ and $\mathbf{L}_{s+1}^{\prime}$ as shown in Figure 7. By the induction hypothesis, the oriented link $\mathbf{L}^{\prime}=\bigcup_{i \neq s} \mathbf{L}_{i}^{\prime}$ is isotopic in the complement of $L^{\prime}$ to a link which is Legendrian with respect to $\widetilde{\zeta_{0}^{\prime}}$ and satisfies conditions (b) and (c) of the statement. Therefore, without loss of generality we may assume $\mathbf{L}^{\prime}$ to be a Legendrian link $\mathcal{L}^{\prime}=\bigcup_{i \neq s} \mathcal{L}_{i}^{\prime}$, with each of its components having contact framing equal to the canonical framing, and the rotation numbers with respect to the section $v^{\prime}=\left(\varphi^{\prime}\right)_{*}^{-1}(\tau)$ satisfying the stated relations (c).

Since any two sufficiently small Legendrian arcs in a contact three-manifold are contact isotopic (see e.g. [7]), without loss of generality we may assume that (i) there is a contactomorphism $g$ between the dotted three-ball $B$ in Figure 7 and a ball centered at the origin of $\mathbb{R}^{3}$ endowed with the standard contact structure $\{d z+x d y=0\}$ and (ii) the intersection of $B$ with $\mathcal{L}_{s-1}^{\prime}$ and $\mathcal{L}_{s+1}^{\prime}$ is sent by the contactomorphism onto two horizontal arcs sitting in the $y z$-plane, one above and the other below the $x y$-plane, as in the left-hand side of Figure 8 . The righthand side of Figure 8 describes, in the language of front projections (cf. [11]), how to perform a Legendrian band connected sum of $\mathcal{L}_{s-1}^{\prime}$ and $\mathcal{L}_{s+1}^{\prime}$. The result is a Legendrian knot $\mathcal{L}_{s}^{\prime \prime}$ smoothly isotopic to $\mathbf{L}_{s}^{\prime \prime}$ in $\mathcal{C}$. Using the standard formula computing the Thurston-Bennequin number of a Legendrian knot from its front projections, it is easy to check that $\mathcal{L}_{s}^{\prime \prime}$ has contact framing equal to its canonical framing minus one. On the other hand, the diffeomorphism $\psi^{-1}$ sends the canonical framing of $\mathcal{L}_{s}^{\prime \prime}$ to the canonical framing plus one, while the canonical framings of 

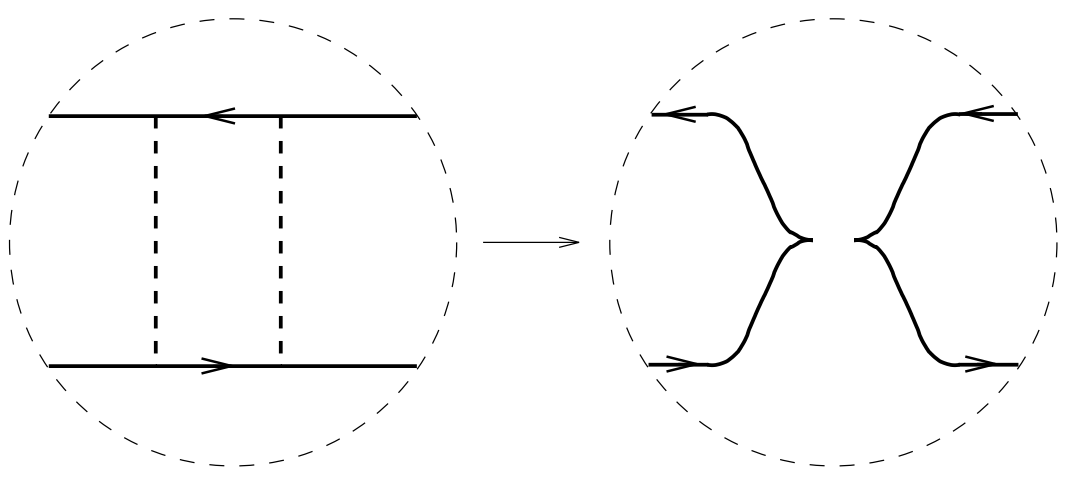

FiguRE 8. The Legendrian band connnected sum

$\mathcal{L}_{i}^{\prime}$, for $i \neq s$, are sent by $\psi^{-1}$ to the canonical framings. This implies that each component of $\mathcal{L}=\psi^{-1}\left(\mathcal{L}^{\prime} \cup \mathcal{L}_{s}^{\prime}\right)$ has canonical framing equal to the contact framing with respect to $\widetilde{\zeta}_{0}=\psi^{-1}\left(\widetilde{\zeta}_{0}^{\prime}\right)$, concluding the proof of (a) and (b) when $s \neq k$.

To prove (c) when $s \neq k$, let $v^{\prime}=\left(\varphi_{*}^{\prime}\right)^{-1}(v)$. Induction applied to the oriented Legendrian link $\mathcal{L}^{\prime}$ inside $N\left(\mathbf{n}^{\prime}\right)$ gives the relations:

$$
\operatorname{rot}_{v^{\prime}}\left(\mathcal{L}_{i-1}^{\prime}\right)+\operatorname{rot}_{v^{\prime}}\left(\mathcal{L}_{i+1}^{\prime}\right)-n_{i} \operatorname{rot}_{v^{\prime}}\left(\mathcal{L}_{i}^{\prime}\right)=\left\{\begin{aligned}
1, & i=1, \\
0, & 1<i<k, \quad i \neq s, s \pm 1 \\
-1, & i=k
\end{aligned}\right.
$$

and

$$
\operatorname{rot}_{v^{\prime}}\left(\mathcal{L}_{s-2}^{\prime}\right)+\operatorname{rot}_{v^{\prime}}\left(\mathcal{L}_{s+1}^{\prime}\right)-\left(n_{s-1}-1\right) \operatorname{rot}_{v^{\prime}}\left(\mathcal{L}_{s-1}^{\prime}\right)= \begin{cases}1, & s=2 \\ 0, & 2<s<k\end{cases}
$$

$$
\operatorname{rot}_{v^{\prime}}\left(\mathcal{L}_{s-1}^{\prime}\right)+\operatorname{rot}_{v^{\prime}}\left(\mathcal{L}_{s+2}^{\prime}\right)-\left(n_{s+1}-1\right) \operatorname{rot}_{v^{\prime}}\left(\mathcal{L}_{s+1}^{\prime}\right)=\left\{\begin{aligned}
0, & 1<s<k-1, \\
-1, & s=k-1 .
\end{aligned}\right.
$$

On the other hand, by Figure 8 and the formula for the rotation number of an oriented Legendrian knot in terms of its front projections [11], we have

$$
\operatorname{rot}_{v^{\prime}}\left(\mathcal{L}_{s-1}^{\prime}\right)+\operatorname{rot}_{v^{\prime}}\left(\mathcal{L}_{s+1}^{\prime}\right)-\operatorname{rot}_{v^{\prime}}\left(\mathcal{L}_{s}^{\prime \prime}\right)=0 .
$$

Now set $\mathcal{L}_{i}=\psi^{-1}\left(\mathcal{L}_{i}^{\prime}\right)$ for $i \neq s$, and $\mathcal{L}_{s}=\psi^{-1}\left(\mathcal{L}_{s}^{\prime \prime}\right)$. The relations (5.3), (5.4), (5.5) and (5.6) provide, when pulled-back via $\psi$, the stated relations for $\mathcal{L}=\bigcup_{i} \mathcal{L}_{i}$. This concludes the proof when $s \neq k$.

When $s=k$ the argument is similar but simpler. The main difference is that Figure 8 should be replaced with Figure 9 . The rest of the argument is essentially the same, so we omit the details.

Corollary 5.2. Let $p>q \geq 1$ be coprime integers with $\frac{p}{p-q}=\left[b_{1}, \ldots, b_{k}\right]$, and let $\mathbf{n}=\left(n_{1}, \ldots, n_{k}\right) \in \mathbf{Z}_{p, q}$. Fix a diffeomorphism $\varphi$ as in (5.2), and let $\mathcal{L}=\bigcup_{i} \mathcal{L}_{i}$ be an oriented Legendrian link as in Theorem 5.1. Let $\widetilde{\mathcal{L}} \subset\left(N(\mathbf{n}), \widetilde{\zeta}_{0}\right)$ be a Legendrian link consisting of $b_{i}-n_{i}$ distinct Legendrian push-offs of each component $\mathcal{L}_{i}$ of $\mathcal{L}$, for $i=1, \ldots, k$. Then, $W_{p, q}(\mathbf{n})$ carries a Stein structure obtained by performing 


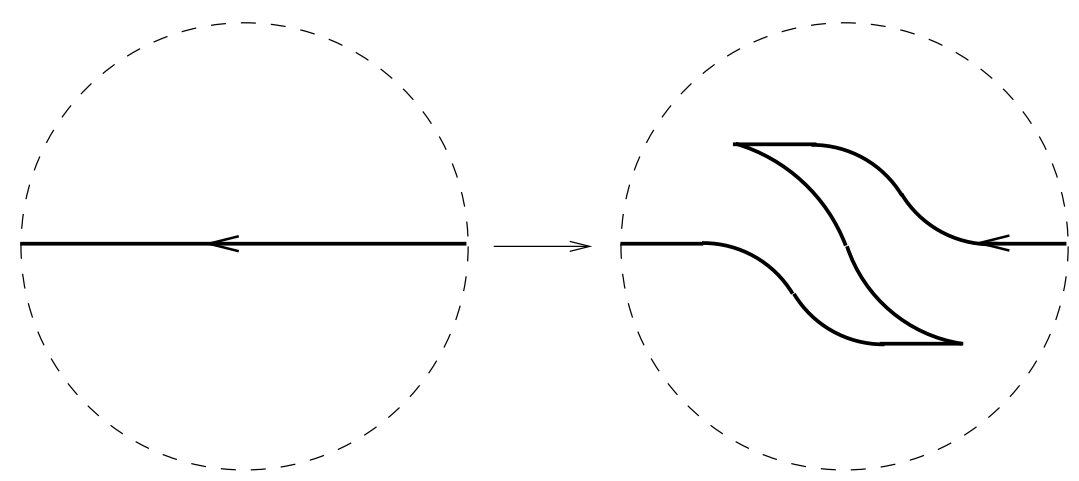

Figure 9. The case $s=k$

Legendrian surgery along the Legendrian link

$$
\varphi(\widetilde{\mathcal{L}}) \subset\left(S^{1} \times S^{2}, \zeta_{0}\right) .
$$

Proof. The statement follows immediately from Theorem 5.1 using Legendrian surgery $[4,11]$.

\section{Contact structures on $\partial W_{p, q}(\mathbf{n})$}

In this section we prove Theorem 1.1(2). The main ingredient of the proof will be the following result.

Theorem 6.1. Let $p>q \geq 1$ be coprime integers and $\mathbf{n} \in \mathbf{Z}_{p, q}$. Let $J$ be a Stein structure on $W_{p, q}(\mathbf{n})$ constructed as in Corollary 5.2, and let $\omega$ be a symplectic form on $W_{p, q}(\mathbf{n})$ compatible with $J$. Then, $\left(W_{p, q}(\mathbf{n}), \omega\right)$ is a symplectic filling of $\left(L(p, q), \bar{\xi}_{\mathrm{st}}\right)$.

Given a Stein structure $J$ on $W_{p, q}(\mathbf{n})$, the distribution

$$
\xi=T \partial W_{p, q}(\mathbf{n}) \cap J T \partial W_{p, q}(\mathbf{n})
$$

of complex lines tangent to the boundary is a contact structure. Therefore, if $\omega$ is a symplectic form on $W_{p, q}(\mathbf{n})$ compatible with $J$, then $\left(W_{p, q}(\mathbf{n}), \omega\right)$ is a symplectic filling of $\left(\partial W_{p, q}(\mathbf{n}), \xi\right)$. We shall establish Theorem 6.1 by proving that if $J$ is constructed as in Corollary 5.2, then the contact three-manifold $\left(\partial W_{p, q}(\mathbf{n}), \xi\right)$ is isomorphic to $\left(L(p, q), \bar{\xi}_{\mathrm{st}}\right)$.

The contact structure $\xi$ is tight [6]. Therefore, in view of the classification of the tight contact structures on $L(p, q)[9,14]$, to show that $\left(\partial W_{p, q}(\mathbf{n}), \xi\right)$ and $\left(L(p, q), \bar{\xi}_{\mathrm{st}}\right)$ are isomorphic it suffices prove that, after a suitable identification $\partial W_{p, q}(\mathbf{n})=L(p, q), \xi$ and $\bar{\xi}_{\text {st }}$ induce the same $\operatorname{Spin}^{c}$ structure on $L(p, q)$, i.e. are homotopic as two-plane fields in the complement of a point. In order to do this we shall use Gompf's $\Gamma$-invariant.

Let $M$ be a closed, oriented three-manifold and denote by $\mathcal{S}(M)$ the set of spin structures on $M$. Given an oriented two-plane field $\xi$ on a $M$, Gompf [11] defines a map

$$
\Gamma(\xi, \cdot): \mathcal{S}(M) \rightarrow H_{1}(M ; \mathbb{Z})
$$

which depends only on the homotopy class $[\xi]$. Moreover, reversing the orientation of $M$ reverses the sign of $\Gamma(\xi, s)$ for fixed $s$, and fixing $q \in M, \Gamma(\cdot, s)$ classifies oriented two-plane fields on $M \backslash\{q\}$ up to homotopy. 
As shown in [11, Theorem 4.12], it is possible to compute $\Gamma$ when $\xi$ is the distribution of complex lines tangent to the boundary of an almost complex fourmanifold. We will need a slight generalization of that result.

Let $X^{*}$ be a smooth four-manifold obtained by attaching two-handles to $B^{4}$ along a framed link $\Lambda \subset S^{3}$. Let $\Lambda_{1} \subset \Lambda$ be a sublink, and let $X_{1}^{*} \subset X^{*}$ be the submanifold obtained by attaching the two-handles corresponding to $\Lambda_{1}$. We may think of $X^{*}$ as obtained by attaching two-handles along $\Lambda_{2}=\Lambda \backslash \Lambda_{1}$ to the boundary of $X_{1}^{*}$. Suppose that the boundary of $X_{1}^{*}$ is a connected sum of $S^{1} \times S^{2}$ 's, so that $\partial X_{1}^{*}=\partial X_{1}$, where $X_{1}$ is obtained by attaching one-handles to $B^{4}$. Then, the smooth four-manifold $X=X_{1} \cup\left(X^{*} \backslash X_{1}^{*}\right)$ is well-defined because every selfdiffeomorphism of $\partial X_{1}$ extends to $X_{1}$. Suppose that $X$ carries an almost complex structure $J$, and denote by $\xi$ the distribution of complex lines tangent to $\partial X$. Fix a complex trivialization $\tau$ of $T X$ over $X_{1}$. Restricting to $\partial X_{1}, \tau$ determines a Spin structure $s_{0}$ on $\partial X_{1}$, and since the boundaries of $X_{1}$ and $X_{1}^{*}$ are identified, there is a canonically associated characteristic sublink $\Lambda_{0} \subset \Lambda_{1}$ representing the Poincaré dual to the second Stiefel-Whitney class of $T X_{1}^{*}$ relative to $s_{0}$ [13]. Similarly, to any Spin structure $s$ on $\partial X=\partial X^{*}$ one can canonically associate a characteristic sublink $\Lambda(s) \subset \Lambda$. Choose an orientation for $\Lambda$, let $K_{1}, \ldots, K_{n}$ be its components and let $\alpha_{1}, \ldots, \alpha_{n} \in H_{2}\left(X^{*} ; \mathbb{Z}\right)$ be the corresponding two-homology classes. The following statement can be proved by an almost word-for-word repetition of the proof of [11, Theorem 4.12].

Theorem 6.2 ([11]). The Poincaré dual to $\Gamma(\xi, s)$ is equal to the restriction of the class $\rho \in H^{2}\left(X^{*} ; \mathbb{Z}\right)$ determined by the evaluations:

$$
\left\langle\rho, \alpha_{i}\right\rangle=\frac{r_{i}+\operatorname{lk}\left(K_{i}, \Lambda_{0}+\Lambda(s)\right)}{2}, \quad i=1, \ldots, n,
$$

where $r_{i}$ is equal to zero if $K_{i} \subset \Lambda_{1}$, and to the integer obstruction to extending the trivialization $\tau$ over the corresponding two-handle if $K_{i} \subset \Lambda_{2}$.

Remark 6.3. (a) Theorem 6.2 reduces to [11, Theorem 4.12] when $X_{1}$ is endowed with a standard Stein structure, $X$ is obtained by Legendrian surgery along a Legendrian link $\Lambda_{2}$ in "standard form", and the restriction of $\tau$ to $\partial X_{1}$ is induced by the vector field " $\frac{\partial}{\partial x}$ " (cf. [11]). In this case each component $K_{i}$ of the oriented link $\Lambda_{2}$ has a well-defined rotation number - the relative winding number of an oriented tangent vector to $K_{i}$ with respect to $\frac{\partial}{\partial x}$ - which coincides with the number $r_{i}$ as defined above.

(b) As observed in [11], after the statement of Theorem 4.12, replacing $\Lambda_{0}+\Lambda(s)$ in the formula by any smooth one-cycle carried by $\Lambda$ and agreeing with $\Lambda_{0}+\Lambda(s)$ modulo 2 does not change the restriction of $\rho$. Thus, after such a replacement Theorem 6.2 still holds.

(c) Theorem 6.2 applies to another particular case, i.e. when $\Lambda_{1}=\emptyset$ and $X_{1}^{*}=$ $X_{1}=B^{4}$. In this case $\Lambda_{0}=\emptyset$ and for every $K_{i} \subset \Lambda_{2}$ we have $r_{i}=\left\langle c_{1}(J), \alpha_{i}\right\rangle$.

We are going to apply Theorem 6.2 when the link $\Lambda$ is the framed link $L \cup € \subset S^{3}$ of Figure 2, with $\Lambda_{1}=L$ and $\Lambda_{2}=\mathrm{E}$, respectively, the "thin" and the "thick" link. We choose an orientation for $\Lambda$ as follows. Represent $L \cup \mathrm{L}$ as in Figure 5. Orient $\mathrm{E}$ as shown in the picture. Orient $L$ so that each of its components has linking number +1 with one of the corresponding thick meridians shown in Figure 5 .

In this case $X_{1}$ is $S^{1} \times D^{3}$ and $X$ is $W_{p, q}(\mathbf{n})$. Let $s$ be a Spin structure on $\partial W_{p, q}(\mathbf{n})$ determined by a characteristic sublink $\Lambda(s) \subset \Lambda$. Observe that $s$ is 
uniquely specified by a sequence $\left(s_{1}, \ldots, s_{k}\right)$, where $s_{i} \in\{0,1\}$ is equal to 1 if the $i$-th component of $L$ belongs to $\Lambda(s)$ and to 0 otherwise. In fact, since $\Lambda(s)$ is characteristic, it is easy to check that the components of $\mathrm{E}$ belonging to $\Lambda(s)$ are determined by the components of $L$ belonging to $\Lambda(s)$.

Denote by $\mu_{i} \in H_{1}\left(\partial W_{p, q}(\mathbf{n}) ; \mathbb{Z}\right), i=1, \ldots, k$, homology classes corresponding to positively oriented meridians of the components of $L$. The classes $\left\{\mu_{i}\right\}$ generate the first homology of $\partial W_{p, q}(\mathbf{n})$ and satisfy the relations

$$
b_{i} \mu_{i}=\mu_{i-1}+\mu_{i+1}, \quad i=1, \ldots, k,
$$

where $\mu_{i}$ is to be interpreted as the zero class for $i<0$ and $i>k$.

Proposition 6.4. Let $p>q \geq 1$ be coprime integers, and let $\mathbf{n} \in \mathbf{Z}_{p, q}$. Let $J$ be a Stein structure on $W_{p, q}(\mathbf{n})$ constructed as in Corollary 5.2, and let $\xi$ be the contact structure induced on the boundary. Let $s$ be the Spin structure on $\partial W_{p, q}(\mathbf{n})$ determined by the sequence $\left(s_{1}, \ldots, s_{k}\right)$, and set $s_{i}=0$ for $i<1$ and $i>k$. Then,

$$
\operatorname{PD} \Gamma(\xi, s)=\sum_{i=1}^{k} \frac{s_{i-1}+s_{i+1}+b_{i}\left(1-s_{i}\right)}{2} \mu_{i}-\sum_{i=2}^{k} \mu_{i} .
$$

Proof. Let $W^{*}$ be the four-manifold with boundary obtained by attaching twohandles to $B^{4}$ according to the framed link $\Lambda=L \cup \mathbf{L}$ of Figure 2. According to Theorem 5.1, $\mathbf{L}$ is isotopic in the complement of $L$ to a link $\mathcal{L}$, Legendrian with respect to the contact structure $\widetilde{\zeta}_{0}$.

Let $s_{0}$ be the unique spin structure on $S^{1} \times S^{2}$ which extends over a two-handle attached along $S^{1} \times\{p\}$ if and only if the corresponding framing is even. Fix a diffeomorphism $\varphi$ as in (5.2), and let $\tilde{s}_{0}$ be the pull-back of $s_{0}$ under $\varphi$. Let $L_{0} \subset L$ be the characteristic sublink corresponding to $\tilde{s}_{0}$.

Let $\alpha_{1}, \ldots, \alpha_{k} \in H_{2}\left(W^{*} ; \mathbb{Z}\right)$ be the homology classes determined by the components $L_{1}, \ldots, L_{k}$ of $L$, and let $\left\{\beta_{i}^{j_{i}} \mid i=1, \ldots, k, j_{i}=1, \ldots, b_{i}-n_{i}\right\} \subset H_{2}\left(W^{*} ; \mathbb{Z}\right)$ be the classes determined by the components $\mathcal{L}_{i}^{j_{i}}$ of $\mathcal{L}$. Let $L(s) \subset \Lambda$ be the characteristic sublink corresponding to the Spin structure $s$ determined by the sequence $\left(s_{1}, \ldots, s_{k}\right)$. Then, by Theorem $6.2, \operatorname{PD} \Gamma(\xi, s)$ is equal to the restriction to $\partial W^{*}=\partial W_{p, q}(\mathbf{n})$ of the class $\rho \in H^{2}\left(W^{*} ; \mathbb{Z}\right)$ determined by the values:

$$
\begin{aligned}
\left\langle\rho, \alpha_{i}\right\rangle & =\frac{1}{2} \operatorname{lk}\left(L_{i}, L_{0}+L(s)\right), \\
\left\langle\rho, \beta_{i}^{j_{i}}\right\rangle & =\frac{1}{2}\left(r_{i}+\operatorname{lk}\left(\mathcal{L}_{i}^{j_{i}}, L_{0}+L(s)\right)\right) .
\end{aligned}
$$

Notice that

$$
\frac{1}{2}\left(r_{i}+\operatorname{lk}\left(\mathcal{L}_{i}^{j_{i}}, L_{0}+L(s)\right)\right)=\frac{1}{2}\left(r_{i}+\operatorname{lk}\left(\mathcal{L}_{i}, L_{0}+L(s)\right)\right)
$$

for every $j_{i}=1, \ldots, b_{i}-n_{i}$, where $\mathcal{L}_{i}$ is the $i$-th component of the link $\mathcal{L}$ given in Theorem 5.1. Setting

$$
C_{i}=\frac{1}{2} \operatorname{lk}\left(L_{i}, L_{0}+L(s)\right), \quad D_{i}=\frac{1}{2}\left(r_{i}+\operatorname{lk}\left(\mathcal{L}_{i}, L_{0}+L(s)\right)\right),
$$

we have:

$$
\operatorname{PD} \Gamma(\xi, s)=\sum_{i=1}^{k}\left(C_{i}+D_{i}\left(b_{i}-n_{i}\right)\right) \mu_{i}
$$


Observe that when $b_{i}=n_{i}$ there is no component $\mathcal{L}_{i}^{j_{i}}$, but the number $D_{i}$ is still well-defined. Hence, Equation (6.2) holds in any case. If we extend the definition of $C_{i}$ and $D_{i}$ by setting $C_{i}=D_{i}=0$ for $i<0$ and $i>k$, in view of the relations (6.1) we have:

$$
\begin{aligned}
\operatorname{PD} \Gamma(\xi, s) & =\sum_{i=1}^{k} C_{i} \mu_{i}+\sum_{i=1}^{k} D_{i}\left(\mu_{i-1}+\mu_{i+1}-n_{i} \mu_{i}\right) \\
& =\sum_{i=1}^{k}\left(C_{i}+D_{i-1}+D_{i+1}-n_{i} D_{i}\right) \mu_{i} .
\end{aligned}
$$

The following identities are easy to check:

$$
\begin{gathered}
\operatorname{lk}\left(\mathcal{L}_{i-1}, L_{j}\right)+\operatorname{lk}\left(\mathcal{L}_{i+1}, L_{j}\right)-n_{i} \operatorname{lk}\left(\mathcal{L}_{i}, L_{j}\right)+\operatorname{lk}\left(L_{i}, L_{j}\right)=0, \quad i, j=1, \ldots, k, \\
\operatorname{lk}\left(\mathcal{L}_{i}, L(s)\right)=2 s_{i}-1, \quad i=1, \ldots, k, \\
\operatorname{lk}\left(L_{i}, L(s)\right)=b_{i}-n_{i}+s_{i}\left(2 n_{i}-b_{i}\right)-s_{i-1}-s_{i+1}, \quad i=1 \ldots, k .
\end{gathered}
$$

Here a linking number is to interpreted as zero if there is an index less than 1 or bigger than $k$. In view of Theorem 5.1(c), a simple calculation using the identities above gives:

$$
2\left(C_{i}+D_{i-1}+D_{i+1}-n_{i} D_{i}\right)=\left\{\begin{array}{l}
s_{2}+s_{0}+b_{1}\left(1-s_{1}\right), \quad i=1, \\
s_{i+1}+s_{i-1}+b_{i}\left(1-s_{i}\right)-2, \quad i=2, \ldots, k .
\end{array}\right.
$$

The statement follows immediately substituting these values in (6.3).

Proof of Theorem 6.1. Let $\xi$ be the contact structure on $\partial W_{p, q}(\mathbf{n})$ given by the tangent complex lines. As explained at the beginning of the section, in order to prove the rest of the statement it suffices to show that, after the choice of a suitable identification $\partial W_{p, q}(\mathbf{n})=L(p, q)$, for any Spin structure $s$ on $L(p, q)$ we have:

$$
\Gamma(\xi, s)=\Gamma\left(\bar{\xi}_{\mathrm{st}}, s\right) .
$$

Recall that $\left(L(p, q), \bar{\xi}_{\text {st }}\right)$ is the link of a cyclic quotient singularity. Let $R_{p, q}$ be a regular neighborhood of the exceptional divisor inside the canonical resolution of such a singularity. Then $R_{p, q}$ is diffeomorphic to a plumbing of type $\left(-a_{1}, \ldots,-a_{h}\right)$, with

$$
\left\langle c_{1}\left(R_{p, q}\right), x_{i}\right\rangle=2-a_{i}, \quad i=1, \ldots, h,
$$

where $x_{i} \in H_{2}\left(R_{p, q} ; \mathbb{Z}\right)$ is the obvious generator represented by an embedded rational curve $C_{i}$ with self-intersection $-a_{i}$ (see e.g. [1]). Equations (6.4) follow from the adjunction formula.

If we start with an immersed curve in $\mathbb{C P}^{2}$ which is the union $l_{1} \cup l_{2}$ of two distinct complex lines, we can successively blow up the curve and its proper transforms at points not of $l_{1}$, until we obtain a string $C$ of curves of type $\left(1,1-b_{1},-b_{2}, \ldots,-b_{k}\right)$ inside $\mathbb{C P}^{2} \# N \overline{\mathbb{C P}}^{2}$. As the proof of Lemma 3.7 shows, there is a natural orientation preserving diffeomorphism $\varphi$ between the complement $Z$ of a regular neighborhood $\nu(C)$ of $C$ and $R_{p, q}$. Moreover, since the natural generators of $H_{2}(Z ; \mathbb{Z})$ are complex curves, by the adjunction formula $\varphi$ must preserve the first Chern classes. Since $R_{p, q}$ is simply connected, this implies that $\varphi$ preserves the complex structures up to homotopy. It follows that the distribution of complex lines tangent to $\partial Z$ is homotopic to $\bar{\xi}_{\text {st }}$. 
The oriented three-manifold $\partial \nu(C)$ has a surgery presentation given by a chain of $k+1$ unknots $U_{0}, \ldots, U_{k}$ framed, respectively, $1,1-b_{1}, \ldots,-b_{k}$. A Spin structure on $\nu(C)$ is encoded by a characteristic sublink of $\bigcup_{i=0}^{k} U_{i}$ (see [13, Section 5.7]), which can be identified with a $(k+1)$-tuple $\left(t_{0}, t_{1}, \ldots, t_{k}\right), t_{i} \in\{0,1\}$, by requiring that $U_{j}$ belongs to the sublink if and only if $t_{j}=1$.

Blowing down $U_{0}$ gives an identification

$$
\nu(C)=-L(p, q)=L(p, p-q) .
$$

By looking at the effect of the Kirby move on the Spin structure [13, pp. 190-191], one sees that $t_{0}=1-t_{1}$.

We shall compute

$$
\Gamma_{L(p, q)}\left(\bar{\xi}_{\mathrm{st}}, s\right)=-\Gamma_{L(p, p-q)}\left(\bar{\xi}_{\mathrm{st}}, s\right)
$$

applying Theorem 6.2 to the almost complex four-manifold $\nu(C)$.

Define

$$
b_{i}^{\prime}= \begin{cases}1, & i=0, \\ -b_{1}+1, & i=1, \\ -b_{i}, & i=2, \ldots, k .\end{cases}
$$

By Remark 6.3(b) and (c), we can apply Theorem 6.2 with $L_{0}=\emptyset$ and the sign of $L(s)$ reversed. There are natural generators

$$
\nu_{0}, \ldots, \nu_{k} \in H_{1}(\partial \nu(C) ; \mathbb{Z})
$$

corresponding to the meridians of $U_{0}, \ldots, U_{k}$, such that

$$
\begin{array}{r}
\operatorname{PD}_{L(p, p-q)}\left(\bar{\xi}_{\mathrm{st}}, s\right)=\sum_{i=0}^{k} \frac{2+b_{i}^{\prime}\left(1-s_{i}\right)-t_{i-1}-t_{i+1}}{2} \nu_{i} \\
=\frac{2+\left(1-t_{0}\right)-t_{1}}{2} \nu_{0}+\frac{2-\left(b_{1}-1\right)\left(1-t_{1}\right)-t_{0}-t_{2}}{2} \nu_{1} \\
+\sum_{i=2}^{k} \frac{2-b_{i}\left(1-t_{i}\right)-t_{i-1}-t_{i+1}}{2} \nu_{i} .
\end{array}
$$

Blowing down all the $(-1)$-unknots in Figure 2 identifies the boundary of $W_{p, q}(\mathbf{n})$ with $L(p, q)=-\partial \nu(C)$. Under this identification a Spin structure $\left(s_{1}, \ldots, s_{k}\right)$ on $\partial W_{p, q}(\mathbf{n})$ corresponds to

$$
\left(t_{0}, t_{1}, \ldots, t_{k}\right)=\left(1-s_{1}, s_{1}, \ldots, s_{k}\right),
$$

and each generator $\mu_{i}$ is sent to $\nu_{i}, i=1, \ldots, k$.

Since $\nu_{0}=-\nu_{1}$ and $t_{0}=1-t_{1}$, Equation (6.5) and Proposition 6.4 give

$$
\operatorname{PD} \Gamma_{L(p, q)}\left(\bar{\xi}_{\mathrm{st}}, s\right)=\operatorname{PD} \Gamma_{L(p, q)}(\xi, s) .
$$

This concludes the proof.

The following is a restatement of Theorem 1.1(2).

Corollary 6.5. For every $\mathbf{n} \in \mathbf{Z}_{p, q}, W_{p, q}(\mathbf{n})$ carries a symplectic form $\omega$ such that $\left(W_{p, q}(\mathbf{n}), \omega\right)$ is a symplectic filling of $\left(L(p, q), \bar{\xi}_{\mathrm{st}}\right)$. Moreover, the homology group $H_{2}\left(W_{p, q}(\mathbf{n}) ; \mathbb{Z}\right)$ contains no classes of self-intersection -1 . 
Proof. The first part of the statement follows from Theorem 6.1, because a Stein four-manifold with boundary is well-known to carry a symplectic form compatible with the complex structure. Therefore, by Theorems 6.1 and 3.2 there exists a symplectic string $\Gamma$ of type $\left(1,1-b_{1},-b_{2}, \ldots,-b_{k}\right)$ inside a rational symplectic four-manifold $X_{M}$ such that $W_{p, q}(\mathbf{n})$ is orientation preserving diffeomorphic to the complement $X_{M} \backslash \nu(\Gamma)$. If $H_{2}\left(W_{p, q}(\mathbf{n}) ; \mathbb{Z}\right)$ contained a class of square -1 , then by Lemma $4.5 W_{p, q}(\mathbf{n})$ would contain a smooth $(-1)$-sphere. But by Corollary 5.2 $W_{p, q}(\mathbf{n})$ carries a Stein structure, and a Stein four-manifold does not contain embedded (-1)-spheres [18, Proposition 2.2]. This proves the second part of the statement.

\section{The Proof of Theorem 1.1}

Let $p>q \geq 1$ be coprime integers, with $\frac{p}{p-q}=\left[b_{1}, \ldots, b_{k}\right]$, and let $R_{p, p-q}$ be a regular neighborhood of the exceptional divisor in the canonical resolution of the cyclic quotient singularity of type $(p, p-q) . R_{p, p-q}$ is diffeomorphic to a plumbing of 2 -disk bundles over 2 -spheres of type $\left(-b_{1}, \ldots,-b_{k}\right)$, and there is a natural identification $\partial R_{p, p-q}=L(p, p-q)$. By [2], given an orientation preserving diffeomorphism

$$
f: L(p, p-q) \rightarrow L(p, p-q),
$$

the isotopy class of $f$ is uniquely determined by the induced homomorphism

$$
f_{*}: H_{1}(L(p, p-q) ; \mathbb{Z}) \longrightarrow H_{1}(L(p, p-q) ; \mathbb{Z}) .
$$

Moreover, $f_{*}$ can only be multiplication by $1,-1, q$ or $-q$, and if $f_{*}$ is multiplication by $\pm q$, then $q^{2} \equiv 1 \bmod p$. Let $x_{1}, \ldots, x_{k} \in H_{2}\left(R_{p, p-q} ; \mathbb{Z}\right)$ be the natural generators represented by smooth rational curves with self-intersections $-b_{1}, \ldots,-b_{k}$.

Lemma 7.1. Let $p>q \geq 1$ be coprime integers. Let $f: \partial R_{p, p-q} \rightarrow \partial R_{p, p-q}$ be an orientation preserving diffeomorphism such that the induced homomorphism

$$
f_{*}: H_{1}\left(\partial R_{p, p-q} ; \mathbb{Z}\right) \rightarrow H_{1}\left(\partial R_{p, p-q} ; \mathbb{Z}\right)
$$

is multiplication by $q$. Then, $f$ is the restriction of a diffeomorphism

$$
F: R_{p, p-q} \rightarrow R_{p, p-q}
$$

such that

$$
F_{*}\left(x_{i}\right)=x_{k+1-i}, \quad i=1, \ldots, k .
$$

Proof. It suffices to prove that if $q^{2} \equiv 1 \bmod p$, then the four-manifold $R_{p, p-q}$ admits a self-diffeomorphism which preserves the orientation and induces multiplication by $q$ on $H_{1}(L(p, p-q) ; \mathbb{Z})$.

The condition $q^{2} \equiv 1 \bmod p$ is equivalent to

$$
\left(b_{1}, b_{2}, \ldots, b_{k}\right)=\left(b_{k}, b_{k-1}, \ldots, b_{1}\right) .
$$

Therefore, when $q^{2}=1 \bmod p$ the pair $\left(S^{3}, L\right)$ has an involution which can be visualized (after an isotopy of $L$ ) as a $\pi$-rotation around an axis perpendicular to the plane of the picture. The resulting self-diffeomorphism of $R_{p, p-q}$ induces multiplication by $q$ on $H_{1}(L(p, p-q) ; \mathbb{Z})$. To see this, observe that $H_{1}(L(p, p-q) ; \mathbb{Z})$ is generated by the classes $\mu_{1}, \ldots, \mu_{k}$ of oriented meridians of $L_{1}, \ldots, L_{k}$ satisfying Relations (6.1) with $-b_{i}$ instead of $b_{i}$, for $i=1, \ldots, k$. The relations imply that $\mu_{1}$ is a generator, and $\mu_{k}=q \mu_{1}$, and the diffeomorphism sends $\mu_{1}$ to $\mu_{k}$. 
Lemma 7.2. Let $p>q \geq 1$ be coprime integers and $\mathbf{n} \in \mathbf{Z}_{p, q}$. For every integer $r \geq 0$, the smooth four-manifold $\widehat{W}_{p, q}(\mathbf{n})=W_{p, q}(\mathbf{n}) \# r \mathbb{C P}^{2}$ admits an orientation preserving self-diffeomorphism which induces multiplication by -1 on $H_{1}\left(\partial W_{p, q}(\mathbf{n}) ; \mathbb{Z}\right)$.

Proof. Rotation by $\pi$ around a horizontal axis going through each component of the thin link in Figure 2 induces an orientation preserving diffeomorphism

$$
\psi: W_{p, q}(\mathbf{n}) \longrightarrow W_{p, q}(\mathbf{n}) .
$$

Blowing down all the $(-1)$-framed unknots gives in identification

$$
\partial W_{p, q}(\mathbf{n})=L(p, q),
$$

where $L(p, q)$ is viewed as the boundary of $R_{p, p-q}$ with reversed orientation. It follows that $H_{1}\left(\partial W_{p, q}(\mathbf{n}) ; \mathbb{Z}\right)$ is generated by meridians $\mu_{1}, \ldots, \mu_{k}$ of the components of the thin link in Figure 2 satifying Relations (6.1). Since $\mu_{1}$ is a generator and $\psi$ sends $\mu_{1}$ to $-\mu_{1}$, it follows that $\psi$ induces multiplication by -1 on $H_{1}\left(\partial W_{p, q}(\mathbf{n}) ; \mathbb{Z}\right)$. The same conclusion applies to $\widehat{W}_{p, q}(\mathbf{n})$, because we can add $r$ disjoint, unlinked $(-1)$-framed unknots $U_{1}, \ldots, U_{r}$ to Figure 2 and repeat the above argument. The fact that the link $\bigcup_{i} U_{i}$ is invariant up to isotopy under the $\pi$-rotation implies that $\psi$ extends to $\widehat{W}_{p, q}(\mathbf{n})$.

Proof of Theorem 1.1. Theorem 4.1 and Corollary 6.5 give, respectively, (1) and (2). Therefore we only need to prove $(3)$.

If $\left(p^{\prime}, s\right)=(p, r)$ and $\left(q^{\prime}, \mathbf{n}^{\prime}\right)=(q, \mathbf{n})$, then clearly

$$
W_{p, q}(\mathbf{n}) \# r \overline{\mathbb{C P}}^{2} \cong W_{p^{\prime}, q^{\prime}}\left(\mathbf{n}^{\prime}\right) \# s \overline{\mathbb{C P}}^{2} \text {. }
$$

If $\left(p^{\prime}, s\right)=(p, r)$ and $\left(q^{\prime}, \mathbf{n}^{\prime}\right)=(\bar{q}, \overline{\mathbf{n}})$, we have

$$
\frac{p}{p-q}=\left[b_{1}, \ldots, b_{k}\right], \quad \frac{p}{p-q^{\prime}}=\left[b_{1}^{\prime}, \ldots, b_{k}^{\prime}\right]=\left[b_{k}, \ldots, b_{1}\right] .
$$

It follows from the definitions that

$$
W_{p, \bar{q}}(\overline{\mathbf{n}})=W_{p, q}(\mathbf{n})
$$

therefore (7.1) still holds.

Conversely, suppose that (7.1) holds. Then, by Part (2) we have $r=s$. Also, (7.1) implies that $L(p, q) \cong L\left(p^{\prime}, q^{\prime}\right)$, and therefore $p^{\prime}=p$ and either $q^{\prime}=q$ or $q^{\prime}=\bar{q}$.

Let us first suppose that $q^{\prime}=q$. By Theorem 3.2 there exists a rational symplectic four-manifold

$$
X_{M} \cong \mathbb{C P}^{2} \# M \overline{\mathbb{C P}}^{2}
$$

and a symplectic string

$$
\Gamma=C_{0} \cup C_{1} \cup \cdots \cup C_{k} \subset X_{M}
$$

of type

$$
\left(1,1-b_{1},-b_{2}, \ldots,-b_{k}\right)
$$

such that

$$
W_{p, q}(\mathbf{n}) \# r \overline{\mathbb{C P}}^{2}
$$

is orientation preserving diffeomorphic to the complement $X_{M} \backslash \nu(\Gamma)$ of a regular neighborhood of $\Gamma$ in $X_{M}$. The same holds for

$$
W_{p, q}\left(\mathbf{n}^{\prime}\right) \# r \overline{\mathbb{C P}}^{2}
$$


with respect to a symplectic string

$$
\Gamma^{\prime}=C_{0}^{\prime} \cup C_{1}^{\prime} \cup \cdots \cup C_{k}^{\prime} \subset X_{M^{\prime}}
$$

of the same type. Since (7.1) holds, we must have $M=M^{\prime}$ and an induced diffeomorphism

$$
\psi: X_{M} \backslash \nu(\Gamma) \rightarrow X_{M} \backslash \nu\left(\Gamma^{\prime}\right) .
$$

Up to composing $\psi$ with the automorphism of

$$
X_{M} \backslash \nu\left(\Gamma^{\prime}\right) \cong W_{p, q}(\mathbf{n}) \# r \overline{\mathbb{C P}}^{2}
$$

of Lemma 7.2, we may assume that the induced homomorphism $\psi_{*}^{\partial}$ on the first homology of the boundary is either the identity or multiplication by $q$. Observe that

$$
\nu(\Gamma) \cong \nu\left(\Gamma^{\prime}\right) \cong \mathbb{C P}^{2} \# R_{p, p-q} .
$$

By Lemma 7.1, the diffeomorphism induced on the boundary by $\psi$ can be extended to a diffeomorphism $F: R_{p, p-q} \rightarrow R_{p, p-q}$. Then, it is easy to see that $F$ can be extended to a diffeomorphism

$$
\widehat{F}: \mathbb{C P}^{2} \# R_{p, p-q} \rightarrow \mathbb{C P}^{2} \# R_{p, p-q}
$$

such that, under the natural isomorphism

$$
\begin{aligned}
H_{2}\left(\mathbb{C P}^{2} \# R_{p, p-q} ; \mathbb{Z}\right) & =H_{2}\left(\mathbb{C P}^{2} ; \mathbb{Z}\right) \oplus H_{2}\left(R_{p, p-q} ; \mathbb{Z}\right), \\
\widehat{F}_{*} & =\left(\begin{array}{cc}
\operatorname{Id} & 0 \\
0 & F_{*}
\end{array}\right) .
\end{aligned}
$$

It follows that $\psi$ is the restriction of a diffeomorphism

$$
\widehat{\psi}: X_{M} \rightarrow X_{M}
$$

such that if $\psi_{*}^{\partial}$ is the identity, then

$$
\widehat{\psi}_{*}\left[C_{i}\right]=\left[C_{i}^{\prime}\right], \quad i=0, \ldots, k,
$$

and if $\psi_{*}^{\partial}$ is multiplication by $q$, then $q^{2} \equiv 1 \bmod p$ and

$$
\begin{aligned}
\widehat{\psi}_{*}\left[C_{0}\right] & =\left[C_{0}^{\prime}\right], \quad \widehat{\psi}_{*}\left[C_{1}\right]=\left[C_{k}^{\prime}\right]+\left[C_{0}^{\prime}\right], \\
\widehat{\psi}_{*}\left[C_{i}\right] & =\left[C_{k-i+1}^{\prime}\right], i=2, \ldots, k-1, \quad \widehat{\psi}_{*}\left[C_{k}\right]=\left[C_{1}^{\prime}\right]-\left[C_{0}^{\prime}\right] .
\end{aligned}
$$

By Theorem $4.1, \mathbf{n}=\left(n_{1}, \ldots, n_{k}\right)$ is uniquely determined by the homology classes $\left[C_{1}\right], \ldots,\left[C_{k}\right]$, and the same holds for $\mathbf{n}^{\prime}$ and the classes $\left[C_{i}^{\prime}\right]$. Therefore, if Equations (7.2) hold, then

$$
b_{i}-n_{i}=b_{i}-n_{i}^{\prime}, \quad i=1, \ldots, k,
$$

hence $\mathbf{n}^{\prime}=\mathbf{n}$. If $q^{2} \equiv 1 \bmod p$ and Equations (7.3) hold, then

$$
b_{i}-n_{i}=b_{k+1-i}^{\prime}-n_{k+1-i}^{\prime}=b_{i}-n_{k+1-i}^{\prime} \text {, }
$$

therefore $\mathbf{n}^{\prime}=\overline{\mathbf{n}}$. This shows that if $q^{\prime}=q$, then either $\left(q^{\prime}, \mathbf{n}^{\prime}\right)=(q, \mathbf{n})$ or $q^{2} \equiv$ $1 \bmod p$ and $\left(q^{\prime}, \mathbf{n}^{\prime}\right)=(q, \overline{\mathbf{n}})$, i.e. $\left(q^{\prime}, \mathbf{n}^{\prime}\right)=(\bar{q}, \overline{\mathbf{n}})$, which is what we needed to prove.

If $q^{\prime}=\bar{q}$, then, since

$$
W_{p, \bar{q}}\left(\mathbf{n}^{\prime}\right) \# r \overline{\mathbb{C P}}^{2}=W_{p, q}\left(\overline{\mathbf{n}}^{\prime}\right) \# r \overline{\mathbb{C P}}^{2},
$$

by (7.1) and the case $q^{\prime}=q$ just proved, $\left(q, \overline{\mathbf{n}}^{\prime}\right)$ is equal to either $(q, \mathbf{n})$ or (when $\left.q^{2} \equiv 1 \bmod p\right)(\bar{q}, \overline{\mathbf{n}})$, i.e. $\left(q^{\prime}, \mathbf{n}^{\prime}\right)$ is equal to either $(\bar{q}, \overline{\mathbf{n}})$ or $(q, \mathbf{n})$. 


\section{ACKNOWLEDGEMENTS}

The author gratefully acknowledges support and hospitality from the Department of Mathematics of the University of Georgia during the preparation of part of this paper. Warm thanks go to Patrick Popescu-Pampu for his interest in my work and for several remarks and suggestions which allowed me to improve this paper in a few points where it lacked precision, and to András Stipsicz for useful comments.

\section{REFERENCES}

1. W Barth, C Peters, A Van de Ven, Compact complex surfaces, Springer-Verlag, Berlin, 1984. MR749574 (86c:32026)

2. F Bonahon, Difféotopies des Espaces Lenticulaires, Topology 22 (1983) 305-314. MR710104 (85d:57008)

3. J A Christophersen, On the components and discriminant of the versal base space of cyclic quotient singularities, Singularity theory and its applications, Part I (Coventry, 1988/1989), 81-92, Lecture Notes in Math. 1462, Springer, Berlin, 1991. MR1129026 (92j:32127)

4. Y Eliashberg, Topological characterization of Stein manifolds of dimension $>2$, Intern. Journal of Math. 1, No. 1 (1990) 29-46. MR1044658 (91k:32012)

5. Y Eliashberg, On symplectic manifolds with some contact properties, J. Diff. Geom. 33 (1991) 233-238. MR1085141 (92g:57036)

6. Y Eliashberg, Filling by holomorphic discs and its applications, London Math. Soc. Lecture Notes Series 151 (1991) 45-67. MR1171908 (93g:53060)

7. Y Eliashberg, M Fraser, Classification of topologically trivial Legendrian knots. Geometry, topology, and dynamics (Montreal, PQ, 1995), 17-51, CRM Proc. Lecture Notes, 15, Amer. Math. Soc., Providence, RI, 1998. MR1619122 (99f:57007)

8. R Fintushel, RJ Stern, Rational blowdowns of smooth 4-manifolds, J. Differential Geom. 46 (1997), no. 2, 181-235 MR1484044 (98j:57047)

9. E Giroux, Structures de contact en dimension trois et bifurcations des feuilletages de surfaces, Invent. Math. 141 (2000), no. 3, 615-689. MR1779622 (2001i:53147)

10. H Gluck, The embedding of two-spheres in the four-sphere, Bulletin of the Am. Math. Soc. 67 (1961) 586-589. MR0131877 (24:A1724)

11. R E Gompf, Handlebody construction of Stein surfaces, Ann. of Math. (2) 148 (1998), no 2, 619-693. MR1668563 (2000a:57070)

12. M Gromov, Pseudoholomorphic curves in symplectic manifolds, Invent. Math. 82 (1985), no. 2, 307-347. MR809718 (87j:53053)

13. R E Gompf, A I Stipsicz, 4-manifolds and Kirby calculus, Graduate Studies in Mathematics, 20, American Mathematical Society, Providence, RI, 1999. MR1707327 (2000h:57038)

14. K Honda, On the classification of tight contact structures I: Lens spaces, solid tori, and $T^{2} \times I$, Geom. Topol. 4 (2000), 309-368. MR1786111 (2001i:53148)

15. P Lisca, Symplectic fillings and positive scalar curvature, Geom. Topol. 2 (1998), 103-116. MR1633282 (99f:57038)

16. P Lisca, On Lens Spaces and Their Symplectic Fillings, Math. Res. Lett. 11 (2004), no. 1, 13-22. MR2046195 (2004m:57058)

17. P Lisca, On symplectic fillings of lens spaces, talk at the conference Holomorphic Curves and Low-Dimensional Topology, Institute for Advanced Study, March 25-29, 2002.

18. P Lisca, G Matić, Stein 4-manifolds with boundary and contact structures, Top. and its Appl. 88 (1998) 55-66. MR1634563 (99f:57037)

19. D McDuff, The structure of Rational and Ruled symplectic 4-manifolds, J. Am. Math. Soc. 3 (1990) 679-712. MR1049697 (91k:58042)

20. D McDuff, Singularities and positivity of intersections of J-holomorphic curves. With an appendix by Gang Liu. Progr. Math. 117, "Holomorphic curves in symplectic geometry", 191-215, Birkhäuser, Basel, 1994. MR1274930

21. J D McCarthy, J G Wolfson, Symplectic gluing along hypersurfaces and resolution of isolated orbifold singularities, Invent. Math. 119 (1995), no. 1, 129-154. MR1309973 (96b:57040)

22. P Orlik, P Wagreich, Algebraic surfaces with $k^{*}$-action, Acta Math. 138 (1977) 43-81. MR0460342 (57:336) 
23. H Ohta, K Ono, Symplectic fillings of the link of simple elliptic singularities, J. Reine Angew. Math. 565 (2003) 183-205. MR2024651 (2005b:53141)

24. H Ohta, K Ono, Simple singularities and topology of symplectically filling 4-manifold, Comment. Math. Helv. 74 (1999), no. 4, 575-590. MR1730658 (2001b:57060)

25. O Riemenschneider, Deformationen von Quotientensingularitäten (nach zyklischen Gruppen), Math. Ann. 209 (1974) 211-248. MR0367276 (51:3518)

26. J Stevens, On the versal deformation of cyclic quotient singularities, in Singularity theory and its applications, Part I (Coventry, 1988/1989), 302-319, Lecture Notes in Math. 1462, Springer, Berlin, 1991. MR1129040 (93b:14061)

27. M Symington, Symplectic rational blowdowns. J. Diff. Geom. 50 (1998) 505-518. MR1690738 (2000e:57043)

28. J Wahl, Smoothings of normal surface singularities. Topology 20 (1981) 219-246. MR608599 (83h:14029)

Dipartimento di Matematica "L. Tonelli", Università di Pisa, I-56127 Pisa, Italy

E-mail address: lisca@dm.unipi.it 